\title{
Análisis sobre la evolución del desarrollo humano en la península de Yucatán
}

\section{Analysis on the evolution of human development in the Yucatan peninsula}

\author{
Francisco José Zamudio-SÁnCHEZ \\ Margarito Soriano-Montero \\ Pedro Ibarra-Contreras*
}

\begin{abstract}
Human development in Mexico offers a gap between the north and south, the Yucatan Peninsula adds to the differentiation. There are marked differences in the development options of the three states that compose it, if income or services are taken as factors for a decent life. Yucatan falls behind Quintana Roo as a leader while Campeche is closer to the latter. Low incomes in Yucatan determine its lag. High income in Campeche does not correspond to its development. Despite hurricanes, Quintana Roo shows substantial progress in development, explicable by tourism. Yucatan is more equitable in gender and Campeche most inequitable in development. Campeche has the lowest quality, below the national average.
\end{abstract}

Keywords: gender-related development index, public utilities, inequity index, quality.

\section{Resumen}

El desarrollo humano en México muestra un distanciamiento entre las regiones norte y sur; la península de Yucatán se agrega a la diferenciación. Hay diferencias marcadas en las opciones de desarrollo de los tres estados que la componen, sea el ingreso o los servicios el factor para una vida digna. Yucatán a la zaga y Quintana Roo de líder, Campeche más cerca de éste. Bajos ingresos en Yucatán determinan su rezago. El alto ingreso en Campeche no corresponde con su desarrollo. A pesar de los ciclones, Quintana Roo muestra un avance sustancial en desarrollo, explicable por el turismo. Yucatán es más equitativo en género y Campeche más inequitativo en desarrollo. Campeche tiene la menor calidad, y también está por debajo de la nacional.

Palabras clave: desarrollo relativo al género, servicios públicos, índice de inequidad, calidad.

* Universidad Autónoma Chapingo. Correos-e: fjzams@yahoo.com, soriano73@hotmail.com, pibac@hotmail.com. 


\section{Introducción}

En 1990 el Programa de Naciones Unidas para el Desarrollo (PNUD) lanzó el primer Informe sobre desarrollo humano mundial, y desde entonces se publica anualmente. En este informe se propuso una nueva concepción sobre el desarrollo, el llamado desarrollo humano (DH), el cual definen como "proceso por el cual se ofrecen mayores oportunidades para los individuos" (PNUD, 1990: 33).

El concepto de DH se origina de los trabajos de Amartya Sen ${ }^{1}$ quien define al desarrollo como un proceso de expansión de las capacidades humanas, comprendiéndose éstas como aquello que las personas pueden ser o hacer y que finalmente logran, y no a los bienes con los que se dispone ya que la provisión de bienes es necesaria mas no suficiente para la ampliación de esas capacidades (Sen, 2000).

El enfoque de DH propuesto por el PNUD establece que lo que un individuo puede ser o hacer, determina su calidad de vida. En este contexto se propone un índice de desarrollo humano (IDH) como una opción alternativa a las mediciones netamente economicistas, y se calcula a partir de indicadores que reflejan de manera cuantitativa las condiciones prevalecientes de elementos que permiten ampliar las capacidades de los individuos. De acuerdo con el PNUD, los tres aspectos que se toman en cuenta como básicos para la población son: la posibilidad de lograr una vida larga y saludable, el acceso a la educación y la disponibilidad de los recursos necesarios para tener un nivel de vida decoroso. Sin esto, no serían factibles muchas otras oportunidades.

El estudio del DH en términos agregados tiende a ignorar su aspecto distribucional, pues pasa por alto diferencias sistemáticas y potenciales entre los diferentes grupos de personas; en particular, entre hombres y mujeres. En este contexto, el DH relativo al género y su medición resultan de vital importancia al considerar las brechas en el acceso a oportunidades entre hombres y mujeres. El estudio de la equidad de género toma importancia, ya que si no existe se tendrán problemas de distribución y acceso a las oportunidades que frenarán el desarrollo de toda la población.

Por otro lado, el estudio del DH puede arrojar resultados que indiquen que en un municipio dado existen las condiciones mínimas necesarias para que las personas logren desarrollarse plenamente, sin embargo, se debe hacer un análisis más profundo para conocer la distribución que éstas tienen en la población de cada estado. En este contexto, el concepto de inequidad toma un sentido más amplio que en el caso de género,

\footnotetext{
${ }^{1}$ Premio Nobel de Economía 1998, profesor de origen hindú, ha enseñado en el Massachussets Institute of Technology (MIT), en las Universidades de Stanford, Berkeley, Harvard y Cambridge, en Delhi School of Economics y London School of Economics.
} 
no sólo se busca conocer y medir si existen las mismas condiciones para que dos grupos (hombres y mujeres) logren desarrollar plenamente sus capacidades, se busca conocer si todos los individuos, estratos o grupos de la población tienen las mismas oportunidades; por ejemplo, en este estudio se busca saber si existe equidad en las oportunidades de desarrollo de los diferentes estados y municipios, y en caso de que no exista, la intención es medir las distancias entre ellos.

Otro concepto de gran relevancia en el ámbito de estudio del DH es el de calidad. En este trabajo dicho concepto se emplea en el sentido de evaluación y eficiencia de los instrumentos de política económica y social puestos en marcha para mejorar las condiciones y oportunidades de los individuos. La calidad nos indica la capacidad administrativa en una población para transformar el ingreso no sólo en una mayor provisión de bienes, sino en mejores condiciones de vida, en una mayor presencia y mejor distribución de oportunidades, de manera que la población logre acceder a mejores niveles de desarrollo.

En México se han publicado varios trabajos sobre la medición del DH a escalas estatal y municipal, entre otros: De la Torre (1997), Jarque y Medina (1998), Ramírez (1999), Conapo (2001) y García (2002). El primer estudio en México en el que se estima a nivel de desagregación estatal y municipal lo realizó la Universidad Autónoma Chapingo ( $\mathrm{Za}-$ mudio et al., 2001, 2002).

Para estimar el IDH, que aquí llamamos índice de desarrollo humano con producto interno bruto (IDHP), se emplean las variables siguientes: esperanza de vida (Ev), tasa de alfabetización, tasa de niños de seis a 14 años que saben leer y escribir, y producto interno bruto per cápita (РIBP). Esta combinación de variables muestra que el crecimiento económico es necesario mas no suficiente para mejorar el bienestar de una población. Se presenta el índice de desarrollo humano con servicios (IDHS) como una alternativa al IDHO, sustituyendo la información relativa al PIBP por la de los servicios de agua entubada, drenaje y electricidad asociados con sus correspondientes indicadores: Inagu, Indre e Inele. Las diferencias de género y los avances que se tienen en este aspecto son cuestiones que no refleja el IDHP, ya que éste le asigna a cada individuo de una población el logro promedio alcanzado en cada una de las variables que lo conforman. Ante esta situación, Anand y Sen (1995) propusieron la construcción del índice de desarrollo humano relativo al género (DHG), el cual utiliza las mismas variables que el IDHP pero incorpora la desigualdad entre hombres y mujeres. El IDG se desarrolló para mostrar que ninguna sociedad trata de igual manera a las mujeres y a los hombres, y que la igualdad de género no depende del nivel de ingreso de una sociedad (De la Cruz, 2007). $\mathrm{La}$ inequidad que se observa en el $\mathrm{DH}$ es otro factor a tomar en cuenta 
cuando se habla del bienestar de las personas. Para medir la variación en la distribución del desarrollo en una entidad se construyó un índice de inequidad (Inq) para cada variable de interés, las cuales exhibirán la distribución o carencia de la misma de los componentes principales del DH. Por último se analiza la calidad municipal mediante la construcción de curvas de calidad, herramienta de análisis propuesta por Zamudio et al. (2001). Expresa la eficiencia en la transformación del ingreso en mejores condiciones de salud, educación y servicios básicos. Este análisis facilita la comparación de DH entre dos territorios con un mismo ingreso o la comparación de ingresos entre dos territorios con el mismo DH.

La región sur-sureste de México muestra serios rezagos en su desarrollo socioeconómico con respecto a las regiones centro y norte del país. La región península de Yucatán forma parte del sur-sureste y está conformada por las entidades de Campeche, Quintana Roo y Yucatán de acuerdo con la clasificación de Bassols Batalla (1994: 53). El propósito de este trabajo es analizar la evolución del DH de los estados y municipios en la región península de Yucatán, haciendo uso de las medidas comentadas y con base en la metodología detallada (Demyc, 2003).

\section{Resultados}

La posición relativa a escala nacional de las entidades, respecto a los indicadores componentes del IDHP, permite comparar las condiciones de salud, educación e ingreso entre los estados de la República y con ello evaluar los efectos de las políticas sociales y económicas en el desarrollo de la población. Tales contrastes ayudan a situar a la península en el contexto nacional.

En el cuadro 1 se muestran los valores del IDHP y sus componentes. Cada variable está asociada con un indicador, a saber: de esperanza de vida (Inesp), de alfabetización (Inalf), de matriculación (Inmat) e indicador del producto interno bruto per cápita (Inpibp), así como sus respectivos rangos (R) para los estados que conforman la península de Yucatán. Una diferencia positiva entre los rangos de dos mediciones indica el número de posiciones que avanzó la entidad en ese periodo. Es necesario indicar que una diferencia negativa no necesariamente implica una disminución del componente del desarrollo que mide el indicador correspondiente, pero sí es muestra de que se tuvo un crecimiento menor al de otros estados del país. 


\subsection{Esperanza de vida en la región península de Yucatán}

"A pesar de los esfuerzos de política social encaminados a procurar la plena integración de la población a los beneficios del desarrollo, es evidente que millones de personas subsisten en tales condiciones adversas que no les es posible satisfacer siquiera sus necesidades básicas más apremiantes. Esta situación estructural se ha visto agravada por recurrentes crisis económicas, que impactaron de manera negativa e intensa la capacidad adquisitiva de amplios segmentos de la población" (Conapo, 2000: 213).

La salud es uno de los componentes fundamentales del DH y de los derechos humanos. La esperanza de vida al nacer (Ev) se considera una de las variables más reveladoras en cuanto al bienestar social y los logros de una región en lo referente a salud. De acuerdo con el Conapo (2007), la reducción en la mortalidad infantil y el consecuente aumento de la EV experimentado por la población mexicana en los últimos años, se ha debido en gran medida al descenso de las afecciones transmisibles, maternas y perinatales; sin embargo, persisten otras causas de muerte principalmente en menores de un año, como las enfermedades infecciosas y parasitarias (Guerrero y Chiapas son las entidades con mayor número de defunciones infantiles); en menores de cinco años las principales causas de muerte son accidentes, anomalías congénitas, enfermedades infecciosas respiratorias y parasitarias (Zacatecas es el estado con la mayor tasa de mortalidad por accidentes). En la población en edad escolar se mantienen las muertes por anomalías congénitas y tumores malignos, y enfermedades genitourinarias (Aguascalientes y Tlaxcala ocupan los primeros lugares en esta categoría). Entre adolescentes y jóvenes las principales causas de mortalidad son las lesiones intencionales, tumores malignos, trastornos mentales y accidentes (Zacatecas, Baja California Sur y Querétaro ocupan el primer lugar nacional en muertes por accidentes de adolescentes y jóvenes). Los cambios que se han tenido en los niveles de mortalidad en México se reflejan en el aumento de la esperanza de vida al nacer, ya que entre 1995 y 2005 aumentó 2.03 años, al pasar de 72.58 a 74.61 años.

Durante el decenio de análisis, de las 32 entidades que conforman la República Mexicana, 14 mejoraron su clasificación, entre ellas Campeche. En 1995 se ubicaba en la posición siete, con una EV de 74.44 años, superior a la nacional (72.58). El aumento de 1.96 años durante el quinquenio 1995-2000 le fue suficiente para alcanzar el lugar tres, donde se mantuvo hasta 2005, en el cual su expectativa de vida era de 76.18 ańos; llama la atención que para ese mismo ańo Campeche era el estado de la península con el mayor número de centros de salud, aunque también el 
que tenía menos médicos (ss, 2006), haciendo difícil inferir si la atención médica coadyuva en el avance de la Ev.

\section{Cuadro 1}

Península de Yucatán, rangos y crecimientos porcentuales de los componentes del IDHP en los periodos 1995-2000 y 2000-2005

\begin{tabular}{|c|c|c|c|c|c|c|c|c|c|c|}
\hline \multirow[b]{2}{*}{ Nombre } & \multicolumn{2}{|l|}{1995} & \multicolumn{2}{|l|}{2000} & \multicolumn{2}{|l|}{2005} & \multicolumn{2}{|c|}{$\begin{array}{c}\text { Avance en los } \\
\text { rangos }\end{array}$} & \multicolumn{2}{|c|}{$\begin{array}{l}\text { Crecimiento } \\
\text { porcentual }\end{array}$} \\
\hline & Inesp & $\mathrm{R}$ & Inesp & $\mathrm{R}$ & Inesp & $\mathrm{R}$ & $95-0000$ & -05 & $95-00$ & 00-05 \\
\hline Nacional & 0.70967 & & 0.73133 & & 0.74358 & & & & 5.26 & 3.12 \\
\hline Campeche & 0.74067 & 7 & 0.76167 & 3 & 0.76974 & 3 & 4 & 0 & 5.47 & 2.22 \\
\hline Quintana Roo & 0.75750 & 1 & 0.77583 & 2 & 0.77220 & 2 & -1 & 0 & 4.99 & -1.04 \\
\hline \multirow[t]{2}{*}{ Yucatán } & 0.72800 & 13 & 0.75017 & 11 & 0.75780 & 14 & 2 & -3 & 5.6 & 2.04 \\
\hline & Inalf & $\mathrm{R}$ & Inalf & $\mathrm{R}$ & Inalf & $\mathrm{R}$ & & & & \\
\hline Nacional & 0.89258 & & 0.90451 & & 0.91459 & & & & 6.22 & 5.83 \\
\hline Campeche & 0.86075 & 23 & 0.88129 & 23 & 0.89500 & 23 & 0 & 0 & 8.57 & 6.55 \\
\hline Quintana Roo & .90181 & 17 & 0.92407 & 14 & 0.93 & 15 & 3 & -1 & 12.57 & 6.4 \\
\hline \multirow[t]{2}{*}{ Yucatán } & 0.84946 & 25 & 0.87 & 25 & 0.88 & 25 & 0 & 0 & 10.36 & 6.1 \\
\hline & & $\mathrm{R}$ & & $\mathrm{R}$ & & $\mathrm{R}$ & & & & \\
\hline Nacional & 0.85994 & & 0.87280 & & 0.87793 & & & & 5.34 & 2.31 \\
\hline Campe & 0.82431 & 25 & 0.84145 & 28 & 0.84696 & 29 & -3 & -1 & 5.92 & 2.06 \\
\hline Quintana Roo & 0.83728 & 24 & 0.85 & 22 & 0.8 & 15 & 2 & 7 & 8.26 & 8.56 \\
\hline \multirow[t]{2}{*}{ Yucatán } & 0.82104 & 27 & 0.86207 & 21 & 0.86075 & 27 & 6 & -6 & 13.96 & -0.56 \\
\hline & INPIBP & $\mathrm{R}$ & INPIBP & $\mathrm{R}$ & INPIBP & $\mathrm{R}$ & & & & \\
\hline Nacional & 0.71931 & & 0.75229 & & 0.75101 & & & & 8.17 & -0.35 \\
\hline Campeche & 0.80926 & 2 & 0.82262 & 3 & 0.83176 & 3 & -1 & 0 & 4.33 & 3.13 \\
\hline Quintana Ro & 0.80710 & 3 & 0.81743 & 4 & 0.80647 & 6 & -1 & -2 & 3.32 & -3.67 \\
\hline \multirow[t]{2}{*}{ Yucatán } & 0.67666 & 19 & 0.71286 & 18 & 0.71303 & 19 & 1 & -1 & 8.27 & 0.04 \\
\hline & IDHP & $\mathrm{R}$ & IDHP & $\mathrm{R}$ & IDHP & $\mathrm{R}$ & & & & \\
\hline Nacional & 0.77023 & & 0.79252 & & 0.79898 & & & & 6.3 & 1.97 \\
\hline Campeche & 0.79951 & 6 & 0.81743 & 7 & 0.82683 & 6 & -1 & 1 & 5.59 & 3.15 \\
\hline & 0.81497 & 3 & 0.83197 & 3 & & 4 & 0 & -1 & 5.64 & -0.21 \\
\hline Yucatán & 0.74821 & 22 & 0.77812 & 19 & 0.78352 & 20 & 3 & -1 & 7.94 & 1.56 \\
\hline
\end{tabular}

Fuente: Cálculos propios con base en INEGI (1990, 1995, 2000, 2005).

Seis entidades del país no presentaron cambios entre 1995 y 2005, teniendo como particularidad que las posiciones que ganaron (perdieron) en los primeros cinco años, las perdieron (ganaron) en los cinco años subsecuentes. Este grupo de estados tuvo la peculiaridad de que durante los años de análisis se ubicaron por arriba de la posición 14 y sólo Chiapas y el Distrito Federal siempre mostraron ev inferiores a la nacional. A pesar de esta aparente ausencia de cambios, las seis entidades mostraron avances importantes en su EV durante el decenio: 1.84, 1.91, 1.98, 1.99, 
2.33 y $2.33 \%$ para Michoacán, Tabasco, Jalisco, Guanajuato, Chiapas y el Distrito Federal, respectivamente.

Yucatán y Quintana Roo se encuentran entre los 12 municipios que retrocedieron en la clasificación nacional con respecto a la Ev, disminuyendo su ubicación en sólo una posición. En este grupo también destacan Chihuahua, Sonora y Baja California que pasaron de las posiciones 28, 25 y 29 a la 32, 29 y 31, respectivamente, además durante los 10 años se encontraron por debajo de la esperanza de vida promedio del país; las condiciones de violencia en muchas de las ciudades del norte del país son causas en la disminución de su Ev.

Considerando el crecimiento acumulado en el Inesp durante el decenio, se pueden distinguir los estados que tuvieron un mayor crecimiento que el promedio nacional y aquellos que lo hicieron por debajo de dicha referencia. Fueron 12 los estados con mayor crecimiento en ev; destacan Hidalgo (16.70\%), Colima (13.54\%), San Luis Potosí (13.07\%), Tabasco $(12.16 \%)$ y Jalisco (11.97\%). Al inicio del periodo su expectativa de vida era mayor a la del promedio nacional, aun con ello tuvieron incrementos significativos.

Los tres estados de la península de Yucatán mostraron crecimientos porcentuales inferiores a la media nacional, sin embargo, en $1995 \mathrm{su} \mathrm{EV}$ se encontraba por arriba de la longevidad promedio del país, lo cual resultaría normal bajo el supuesto de rendimientos decrecientes. Quintana Roo tuvo el menor incremento, pero al principio del periodo tenía la mayor expectativa de vida del país.

En la figura i se observa cómo la mayoría de los estados de la República se ajustan a una tendencia bien definida, que no es perfectamente lineal debido a oscilaciones naturales, la autonomía de los gobiernos estatales y los resultados de las políticas sociales que estos emprendieron en su territorio. Los estados que en 1995 tenían la peor Ev lograron los mayores incrementos porcentuales en el decenio; solamente Chihuahua, Baja California y Sonora mostraron un comportamiento diferente, ya que a pesar de su mala situación al principio del periodo manifestaron los crecimientos más bajos, teniendo un comportamiento dañino y aparentemente persistente. Las líneas blancas muestran los valores correspondientes a la República Mexicana.

La mayoría de los estados que en 1995 tenían una EV inferior a la nacional, durante los 10 años subsecuentes tuvieron incrementos por debajo de la expectativa nacional, es decir, tenían rezagos y siguieron con ellos. Por otro lado, los estados que al inicio del periodo tenían una EV superior al promedio nacional, incluidos Campeche, Quintana Roo y Yucatán, tuvieron menores incrementos durante el decenio. El análisis de rangos y de crecimiento enmarca estados de la República que sobresalen 


\section{Figura I}

\section{Inesp $1995 v s$. incremento porcentual Inesp 1995-2005}

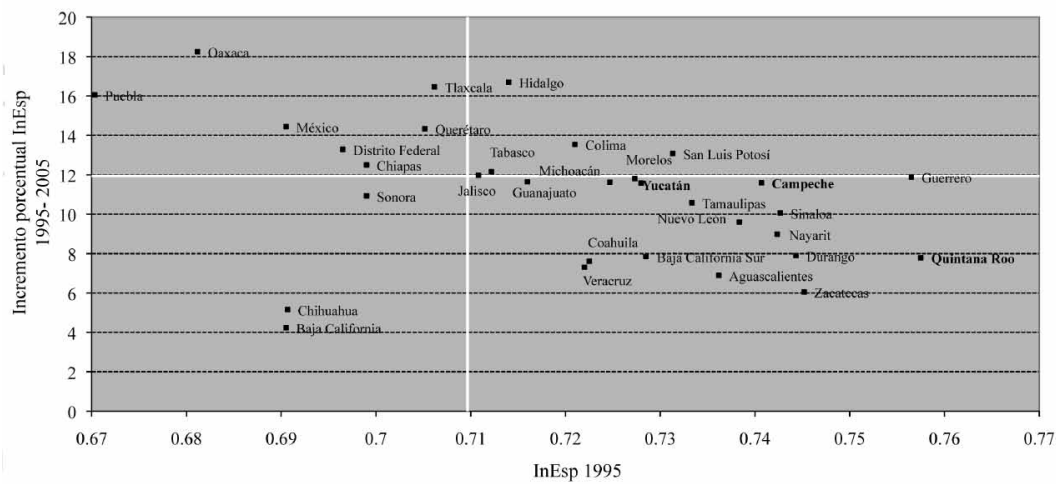

Fuente: Cálculos propios con base en INEGI (1990, 1995, 2000, 2005).

por su condición crítica o favorable; en los primeros se ubican Baja California y Chihuahua, en el segundo grupo se encuentran Hidalgo, Colima y San Luis Potosí, los pertenecientes a la península se encuentran dentro de la regularidad.

Muchos factores se relacionan con la Ev, como la infraestructura y el personal médico, si pertenecen a algún servicio de salud, los fenómenos naturales, entre otros. Algunos aspectos relevantes sobre ellos explican parcialmente los resultados.

En cuanto a infraestructura y personal médico (cuadro 2), tanto en el año 2000 como en el 2005 Campeche se encontraba en una buena posición con respecto al país; mientras que Yucatán estaba en una situación muy cercana a la nacional, logrando avances considerables en casi todos los indicadores. En el año 2000 Quintana Roo tenía una situación superior a la del promedio nacional, pero los retrocesos en todos sus recursos materiales y humanos causaron una gran disminución en sus condiciones para 2005, colocándose por debajo de la situación nacional en camas censables y enfermeras.

En 1995, 2000 y 2005 Quintana Roo fue el estado con las menores tasas de mortalidad por cada 1,000 habitantes, $2.60,2.55$ y 2.69 , muy por debajo del promedio nacional, 4.70, 4.47 y 4.79, respectivamente; durante estos años la mortalidad en Campeche también fue inferior a la del país, con tasas por cada 1,000 habitantes de 3.73, 3.41 y 3.79, ubicándose en 1995 en la posición cinco. En el otro extremo de la escala se encontró el estado de Yucatán, cuya mortalidad promedio fue mayor a la nacional: alcanzó tasas de 5.42, 5.08 y 5.30, pasó de la posición 29 en 1995, a la 30 en los otros años de estudio (ss, 2005). 


\section{Cuadro 2}

Indicadores de recursos materiales y humanos por cada 100,000 habitantes

\begin{tabular}{lrllllllrr}
\hline \multicolumn{1}{c}{ Nombre } & \multicolumn{2}{c}{$\begin{array}{c}\text { Camas } \\
\text { censables }\end{array}$} & \multicolumn{2}{c}{ Consultorios } & \multicolumn{2}{c}{ Médicos } & \multicolumn{2}{c}{ Enfermeras } \\
& 2005 & 2000 & 2005 & 2000 & 2005 & 2000 & 2005 & 2000 \\
Nacional & 57.39 & 57.29 & 45.84 & 42.56 & 104.32 & 90.42 & 134.55 & 123.6 \\
Campeche & 83.8 & 91.92 & 65.71 & 69.72 & 155.46 & 129.26 & 212.6 & 181.75 \\
Quintana Roo & 46.55 & 58.29 & 58.04 & 72.52 & 116.08 & 127.55 & 129.1 & 168.36 \\
Yucatán & 56.15 & 60.75 & 44.9 & 38.03 & 100.72 & 90.41 & 143.72 & 136.92 \\
\hline
\end{tabular}

Fuente: Secretaría de Salud, http://sinais.salud.gob.mx/infraestructura.

La disponibilidad y acceso a los servicios de salud es una probable causa de las condiciones y cambios en la longevidad en los diferentes estados. Entre 2000 y 2005 sólo tres entidades, incluida Quintana Roo, redujeron el porcentaje de población que recibía estos servicios. El motivo de que la mayoría de los estados incrementaran dicha proporción, a pesar de que las principales instituciones [Instituto Mexicano del Seguro Social (IMss) e Instituto de Seguridad y Servicios Sociales de los Trabajadores del Estado (ISSSTE)] redujeron su cobertura en términos porcentuales (INEGI, 2000, 2005), fue la población que se afilió al Seguro Popular (sP), programa creado en 2001 dentro del Plan Nacional de Salud 2001-2006. En Campeche (INEGI, 2001a y 2006a) y Yucatán (INEGI, 2001c, 2006c) disminuyó el número de beneficiados por las instituciones existentes en el ańo 2000 en 32.2 y 19.9 puntos porcentuales, respectivamente, para 2005 el 32 y $15.5 \%$ de la población eran derechohabientes del Seguro Popular, mientras que en Quintana Roo (INEGI, 2001b, 2006b) este programa y el aumento en la cobertura de instituciones de menor peso en el sector salud, como Petróleos Mexicanos (Pemex), la Secretaría de la Defensa Nacional (Sedena) o la Secretaría de Marina (Semar) evitaron que la crisis y poco crecimiento del IMSS e ISSSTE trajeran consigo una caída mucho mayor en cuanto al porcentaje de la población estatal beneficiada. El vacío dejado por las instituciones de salud del país y que llenó el sp tiene sus acotaciones: las evaluaciones que se han hecho del mismo presentan resultados insatisfactorios (Scott, 2006) y hay factores políticos, institucionales, administrativos y metodológicos que invalidan la evaluación del programa (King, 2006), no obstante, la sustitución de derecho por servicios de salud que se acredita al sp no está en discusión, lo que sí lo está es la calidad y forma en que se da la inscripción al mismo.

En la península de Yucatán los huracanes son fenómenos frecuentes y, según la Comisión Nacional del Agua (CNA), ningún otro punto del país es tan propenso a estos fenómenos (Tun y Manzanilla, 2005). En el año 


\section{Cuadro 3}

Población derechohabiente en servicios de salud

\begin{tabular}{llc}
\hline \multicolumn{1}{c}{ Nombre } & Porcentaje de población derechohabiente \\
\hline & 2000 & 2005 \\
Nacional & 40.1 & 46.9 \\
Campeche & 38.4 & 58.3 \\
Quintana Roo & 46.2 & 46.0 \\
Yucatán & 45.1 & 52.8 \\
\hline
\end{tabular}

Fuente: INEGI $(2000,2005)$.

2000 casi cuatro millones de personas se encontraban en zona de riesgo, sin embargo, no se cuenta con evidencias directas que muestren que la presencia de ciclones y los destrozos que estos han causado hayan tenido efecto en la expectativa de vida de los habitantes de la región. Las únicas evidencias son las indirectas y se concentran en el estado de Quintana Roo, el territorio más golpeado del país en la última década. Así, se observan menores recursos humanos y materiales en el sector salud, menos afiliados al servicio y un crecimiento muy pobre en la Ev de sus habitantes.

Otro factor que incide en la ev es el acceso a ciertos servicios públicos, los cuales se tratan en secciones posteriores. Según Kliksberg (2000: 9), el acceso a agua potable, instalaciones sanitarias, alcantarillado y energía eléctrica determinan las distancias entre diversas áreas geográficas en el sector salud.

En el cuadro 4 se presenta el número de personas que para el año indicado tenían una $\mathrm{EV}$ igual o menor al valor correspondiente a la columna "Ańos", o cuyo Inesp era cuando mucho igual al valor de la columna " $\mathrm{x}$ ". Las columnas de incorporaciones absolutas muestran el número de personas desincorporadas (positivos) o incorporadas (negativos) en los periodos de análisis. Por último, en las columnas de incorporaciones porcentuales aparecen los porcentajes de personas incorporadas o desincorporadas a cada categoría durante el quinquenio señalado respecto al total de lo ocurrido en el decenio.

En los estados de la región, la dinámica poblacional y posiblemente las políticas puestas en marcha han traído un aumento paulatino de la cota inferior de la esperanza de vida. Se espera que el rango de la EV vaya disminuyendo con el paso del tiempo, aproximándose a una especie de estado estacionario. En Campeche, para el grupo de EV entre 74.4 y 75.6 años se aprecia una desincorporacion importante en el segundo quinquenio muy por encima de la incorporación existente en los primeros cinco años; además, para el último quinquenio se comenzaron a abandonar las categorías de menos de 78 años en las que se ubicaba la mayoría de la 


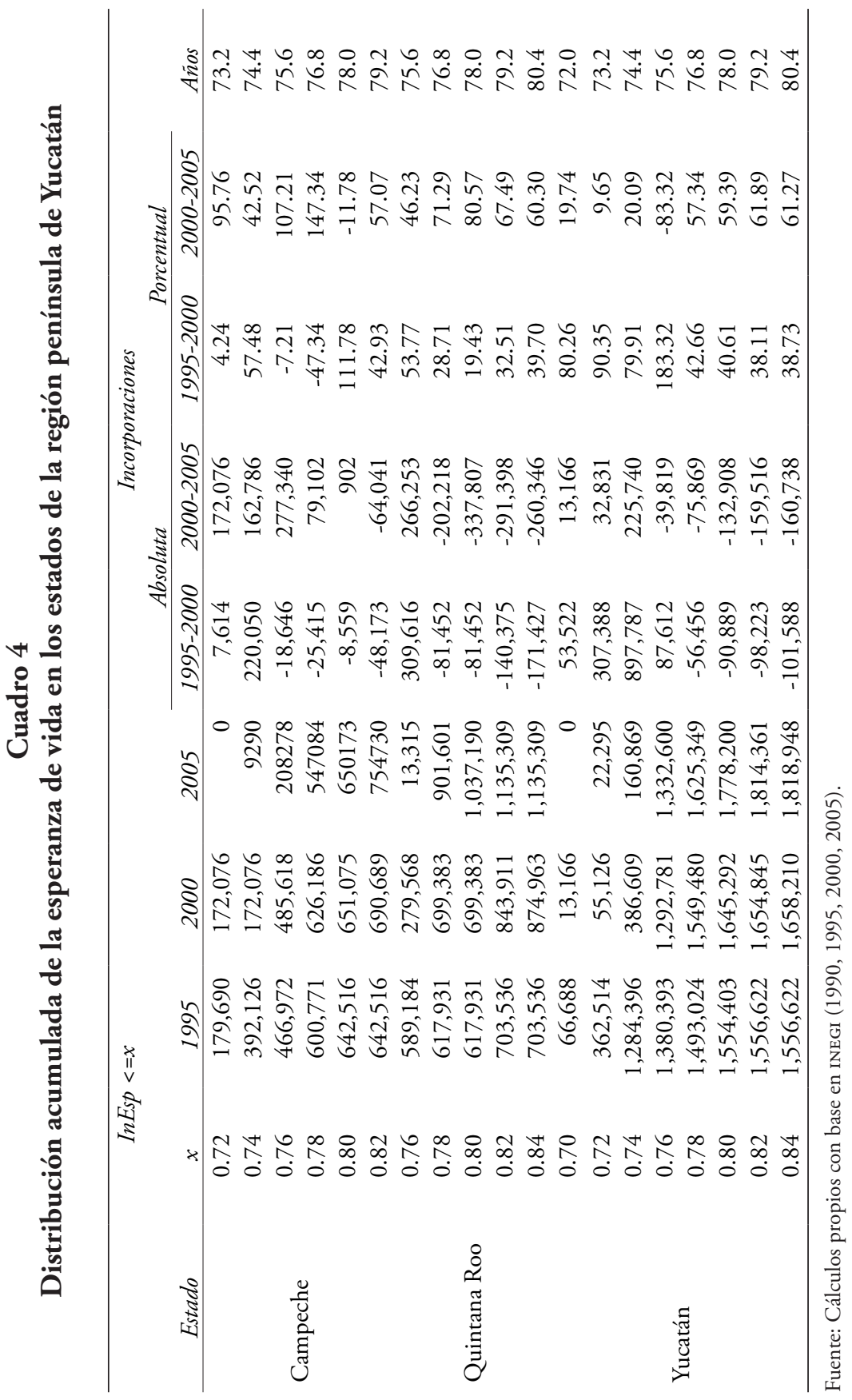


población, ampliando así el número de personas en categorías más altas. En la categoría entre 74.4 y 75.6 años, el estado de Yucatán muestra una desincorporación en el primer periodo, y una incorporación de poco menos de 40,000 personas para el siguiente quinquenio, se tuvo un aumento en el número de personas que conformaban dicha clase entre 2000 y 2005, causado en gran medida por ocho municipios de la entidad que pasaron de la categoría inmediata superior a ésta, entre ellos, Kanasin que en 2005 contaba con 51,174 habitantes. Yucatán es la entidad que se muestra más rezagada en este aspecto en la península, además de mostrar el rango más amplio de la Ev. Quintana Roo es el estado de mayor $\mathrm{EV}$ en la región, pues aquí la categoría más alta (79.2-80.4 años) se vio ampliada durante el último quinquenio en más de 260,000 personas, por arriba de lo ocurrido en Yucatán para la misma categoría, a pesar de que la población quintanarroense era mucho menor.

\subsection{Educación en la región península de Yucatán}

El estudio del perfil educativo en las regiones de México es relevante, ya que contribuye a explicar algunas diferencias socioeconómicas y de desarrollo, como la incorporación al mercado laboral, los ingresos personales o el grado de marginación de las poblaciones (Bracho, 1999). Para el análisis del aspecto educativo se consideran dos indicadores: el alfabetismo en la región y la matriculación infantil, empleando como herramientas de medición el Inalf y el Inmat, respectivamente, mediante los cuales el efecto de la educación en el DH se incorpora al IDHP. En esta sección, al hablar de alfabetización nos referimos a alfabetización en la población adulta (mayor de 14 años).

El nivel educativo de una población es muy importante, y se relaciona directamente con otras características sociales y económicas. Naciones Unidas reconoció que crear poblaciones totalmente alfabetizadas es crucial para lograr la reducción de la pobreza, la mortalidad infantil y la inequidad de género, de este modo lograr sociedades con posibilidades de desarrollar sus capacidades.

En el cuadro 1 se aprecia que la tasa de alfabetización nacional aumentó en el decenio: pasó de 0.89258 a 0.91459 , lo cual indica que cada vez más personas mayores de 14 años son censadas como hábiles para leer y escribir. En este periodo la población del país mayor de 14 años aumentó en cerca de 10 millones de personas, mientras que la población en el mismo grupo de edad en condición de analfabetismo disminuyó en poco más de 400,000.

En el decenio, sólo ocho entidades mejoraron su clasificación, entre ellas Quintana Roo y Querétaro lograron la máxima ganancia de posicio- 
nes, al pasar del lugar 17 al 15 y del 21 al 19, respectivamente. Otros estados que sobresalen en este grupo son Aguascalientes, Coahuila y Sonora con tasas de alfabetización superiores a 94 por ciento.

La educación básica en México - por los niveles que alcanza- no es un atributo dinámico, y la privatización de la misma -aun en el nivel básico declarado obligatorio- producirá tasas de crecimiento más bajas, e incluso negativas.

Nueve estados retrocedieron en la clasificación a escala estatal, de ellos solamente Tabasco y Veracruz se encontraron desde 1995 por debajo de la tasa de alfabetización del país. Baja California, Baja California Sur y Chihuahua retrocedieron un lugar: en 2005 se ubicaron en las posiciones cuatro, seis y ocho.

Entre las 15 entidades que mantuvieron su posición se encontraron Campeche y Yucatán (lugares 23 y 25, respectivamente), además siempre presentaron valores del Inalf menores a los del país, y sus incrementos fueron inferiores a los de Quintana Roo. Considerando el aumento estatal del Inalf, 19 entidades tuvieron crecimientos por arriba del nacional, de ellas Aguascalientes, Coahuila, Colima, Jalisco, Estado de México, Morelos, Quintana Roo, Sinaloa, Sonora, Tamaulipas y Tlaxcala tenían tasas de alfabetización superiores a la nacional al inicio del periodo. Yucatán y Campeche también crecieron más que el promedio nacional.

De los estados que menos crecieron -Veracruz, Guerrero, Michoacán y Oaxaca-, en 1995 tenían valores de Inalf menores a los de la República. En 1995 únicamente Guerrero, Chiapas y Oaxaca tenían tasas de analfabetismo superiores a 20\% (Inalf $<0.8$ ), Chiapas no había logrado superar esta situación para 2005. Persiste el rezago en educación e ingreso, donde la marginación, pobreza, ruralidad y otros atributos se mezclan en una asociación viciosa.

En la figura II se observa que se presenta el mismo fenómeno que en la $\mathrm{EV}$, sin embargo la tendencia es menos pronunciada porque aun cuando la alfabetización no ha sido muy dinámica en el país, las ganancias en longevidad son explicablemente todavía más lentas.

"La UNESCO considera que una nación alcanza la alfabetización universal o casi universal cuando más de $90 \%$ de su población adulta está alfabetizada. Sugiere que con un $70 \%$ el progreso hacia la alfabetización universal será irreversible, porque cualquier persona analfabeta sentirá la necesidad de adaptarse a un medio en el que predominan las personas alfabetizadas" (INEGI, 2002). De acuerdo con los cálculos del Inalf, para 2000 y 2005, 22 estados de la República -incluidos Campeche, Quintana Roo y Yucatán- tenían más de $90 \%$ de su población mayor de 14 años alfabetizada. Ya en 1995, 17 estados se encontraban en esa situación. Contar con una alfabetización universal es posicionarse en la cota inferior 


\section{Figura II}

\section{Inalf $1995 v s$. incremento prcentual Inalf 1995-2005}

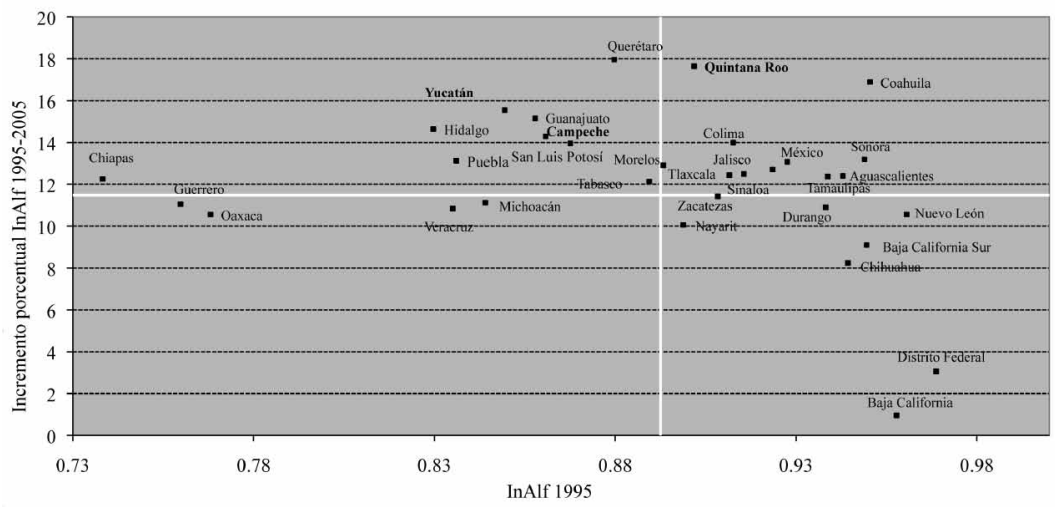

Fuente: Cálculos propios con base en INEGI (1990, 1995, 2000, 2005).

del logro educativo y de las competencias laborales para insertarse en la economía, es una necesidad cuya suficiencia se ubica lejos de una capacidad desarrollada.

Para el análisis de la matrícula infantil se consideró la población entre seis y 14 años de edad que sabe leer y escribir, es un estimador más robusto que aquélla y una medida más directa de la educación. En 1995 en México, $86 \%$ de la población entre seis y 14 años sabía leer y escribir, dicho porcentaje aumentó a aproximadamente $88 \%$ en 2005 . Podemos hacer notar que el aumento de la población en ese grupo de edad para el periodo considerado fue muy bajo, ya que pasó de 19'317,979 a $19 ' 332,243$ por un descenso en la tasa de natalidad en el país. Aun sin crecimiento demográfico, las políticas educativas ofrecen resultados pobres.

En el decenio, 13 estados mejoraron en la clasificación nacional con respecto al Inmat, entre estos destacó Quintana Roo, al pasar de la posición 24 a la 15, presentando la mayor mejora en el periodo al superar en 2005 el Inmat nacional.

Yucatán y otras cuatro entidades no mostraron cambios en su posición, aunque todas se encontraban por encima de la posición 20. Para 1995, únicamente Zacatecas se encontraba por arriba de la matrícula promedio del país, sin embargo, en 2005 todas se ubicaban por debajo de la media. A pesar de que todos los estados avanzaron en este aspecto, los amplios incrementos, principalmente de los estados mencionados en el párrafo anterior, provocaron un alza del Inmat nacional alejando este valor de los indicadores del resto de los estados.

Campeche estuvo entre los 14 estados que retrocedieron, y junto a Oaxaca, Guanajuato y Puebla, al principio del periodo, tenían un Inmat que estaba por debajo de la media nacional; lo cual no habían logrado 
cambiar en 2005. En este conjunto, las caídas en posición fueron causadas por decrementos en el índice para algunos estados, provocando que el Distrito Federal pasara del lugar uno al dos y que Nayarit cayera del ocho al 18.

De las 17 entidades que incrementaron el valor de su Inmat por arriba del crecimiento nacional, $7.47 \%$ (cuadro 1), en 11 de los casos el aumento fue superior a 10\%, ente ellos Quintana Roo (15.84\%); de este grupo Colima, Sinaloa, Hidalgo, Baja California, Aguascalientes, Chihuahua y Durango además de haber mejorado en la clasificación, en 1995 tenían un Inmat por arriba del valor nacional (0.85994).

Coahuila, Nayarit, Distrito Federal, Nuevo León y otras 11 entidades vieron disminuir su tasa de matrícula infantil, donde el caso más drástico fue Nuevo León con una disminución de 22.66\%. En 1995 Oaxaca y Guanajuato se encontraban por debajo de la media nacional, y en 2005 se les unieron Zacatecas y Sonora.

Los estados de la península de Yucatán cumplen con lo esperado, al igual que la mayoría de las otras entidades, sólo resaltaría la mala situación de algunas ya mencionadas como Oaxaca y Guanajuato, y las buenas condiciones de Colima, Hidalgo, Sinaloa, Baja California Sur y Aguascalientes.

En la educación básica, la cantidad de escuelas y maestros en las entidades de la República no revela una gran influencia en los Inmat estatales, ya que en los peores casos se tenían en promedio poco más de 207 alumnos por escuela, como fue el caso del Distrito Federal, y aproximadamente un promedio de 27 alumnos por maestro, como ocurría en Querétaro (SEP, 2006, 2007). Cabe aclarar que no se discute la calidad de la educación ni se toma en cuenta la distribución territorial de las escuelas, maestros y población infantil, pero aparentemente se contaba con los recursos para que fuese de buen nivel, sin embargo, el empobrecimiento en la calidad educativa puede ocurrir, entre otras cosas, por una inadecuada distribución de los recursos materiales y humanos.

Los estados con el mayor porcentaje de población indígena son los que tienen más marginación (empleando el índice de marginación de Conapo), con algunas excepciones como Quintana Roo en la península. En 2000 y 2005 Guerrero, Chiapas y Oaxaca, fueron clasificados con grado de marginación muy alto, y sus respectivos porcentajes de población mayor de cinco años que hablaba una lengua indígena eran 14, 25 y 37 en 2000, y 14, 26 y 35 en 2005, respectivamente. Los porcentajes de población indígena para Nuevo León, Baja California, Distrito Federal y Coahuila, clasificados con marginación muy baja, fueron $0.8,1.4,0.3$ y 


\section{Figura III}

Inmat 1995 vs. incremento porcentual Inmat 1995-2005

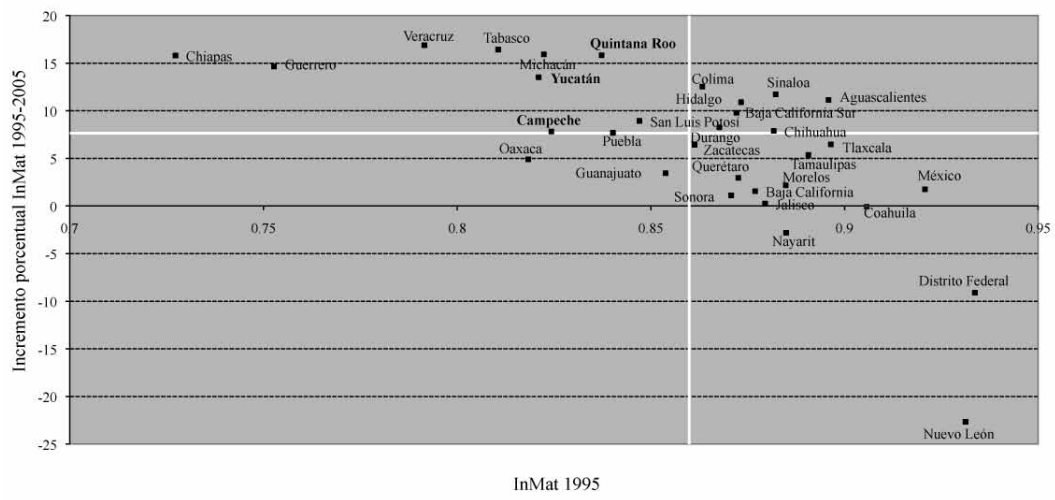

Fuente: Cálculos propios con base en INEGI (1990, 1995, 2000, 2005).

1.5, respectivamente. Al ser la tasa de analfabetismo un componente importante del índice de marginación, se tiene que la población indígena del país es la que más sufre de analfabetismo, lo cual se refleja en los Inalf estatales. En la península de Yucatán fue Quintana Roo el estado que mostró los mayores incrementos y más altas incorporaciones a categorías altas de alfabetismo y matriculación infantil. Hay una tendencia a mejorar los índices educativos en la región.

Los cuadros 6 y 7 muestran que el alfabetismo de los estados de la península se aproxima más rápidamente, que la matriculación infantil, a $100 \%$. Referente a la alfabetización, el estado de Quintana Roo es el que mostró la mejor situación: en 2005 más de la mitad de sus habitantes se ubicaba en municipios donde el porcentaje de la población alfabeta se encontraba entre 94 y 98\%, categoría que a pesar de ser las más alta tuvo las mayores incorporaciones de personas. El estado de Campeche comienza a incorporar gente a las categorías de Inalf superiores a 0.82 , en el periodo $1995-2000$ se observa que la categoría entre 0.86 y 0.90 experimentó una desincorporación de 136,267 personas, haciendo que más de la mitad de la población ascendiera a la categoría más alta.

En 2005 la correlación entre el Inmat y el Inalf en la región fue 0.77781, la más alta entre los componentes del IDHP, lo cual resulta natural ya que mientras existan las políticas, infraestructura y recursos humanos que permitan una alta matriculación infantil se espera que la mayoría de la población mayor de 15 años haya aprendido a leer y escribir a edades tempranas. 


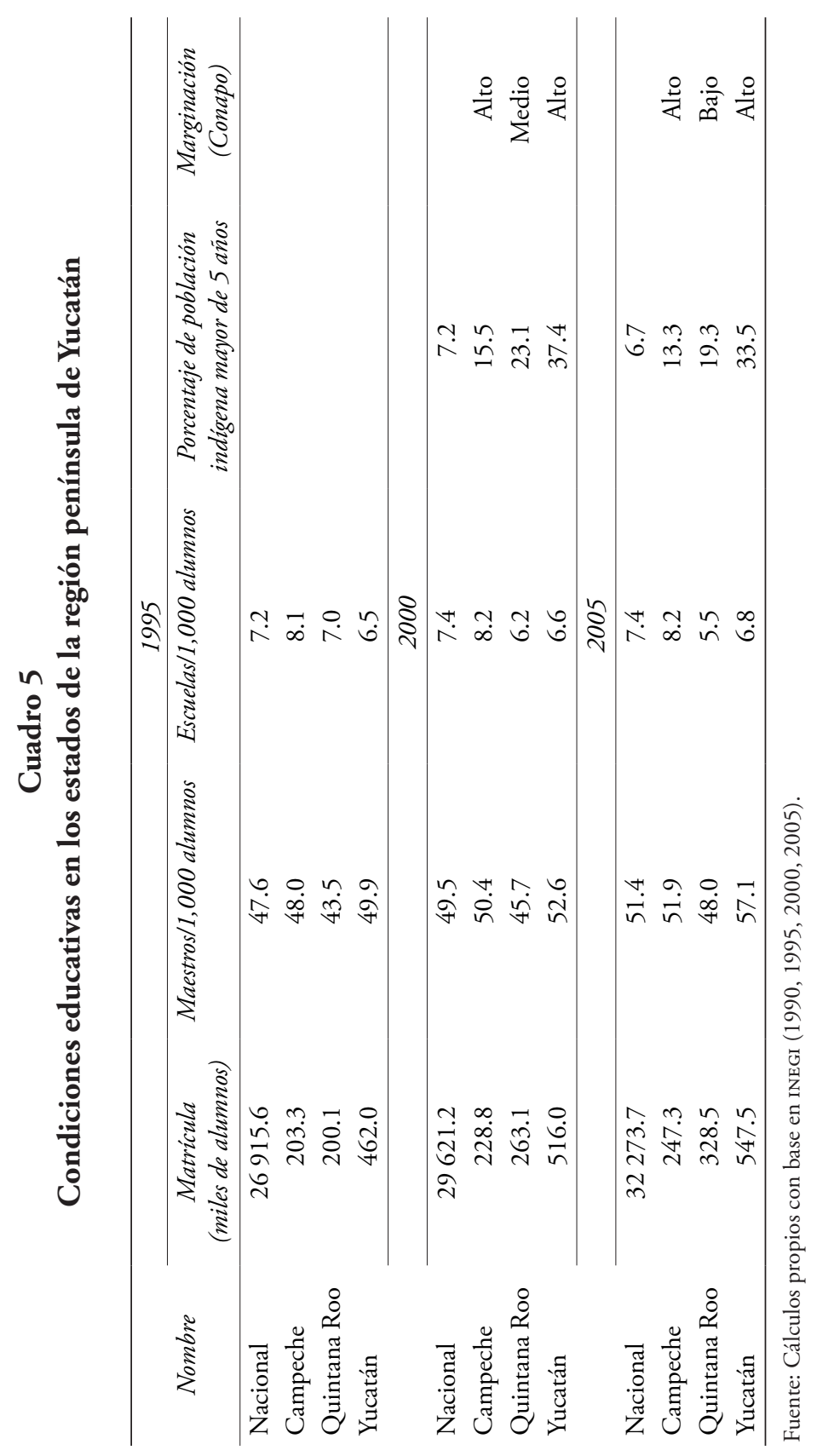




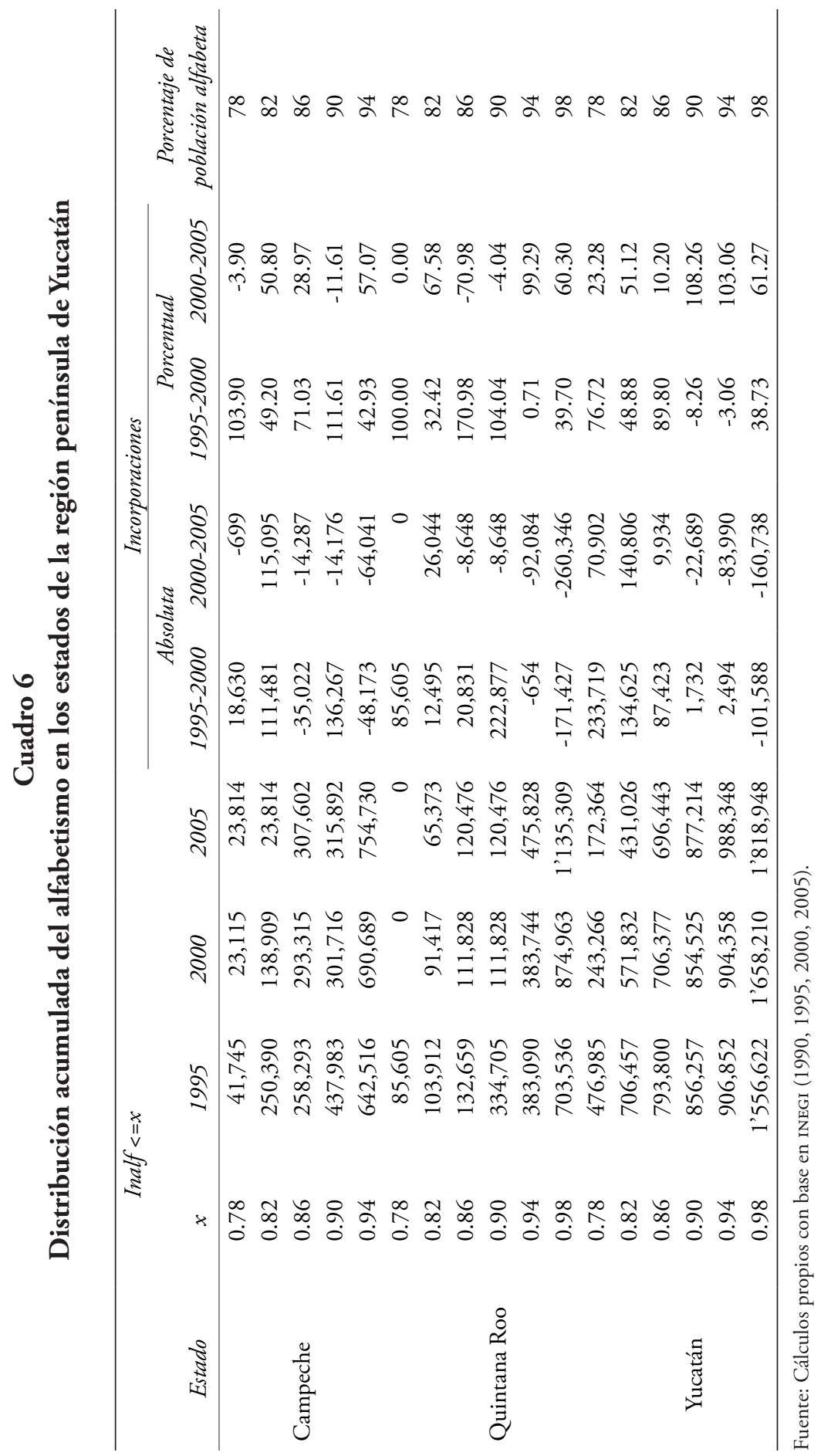




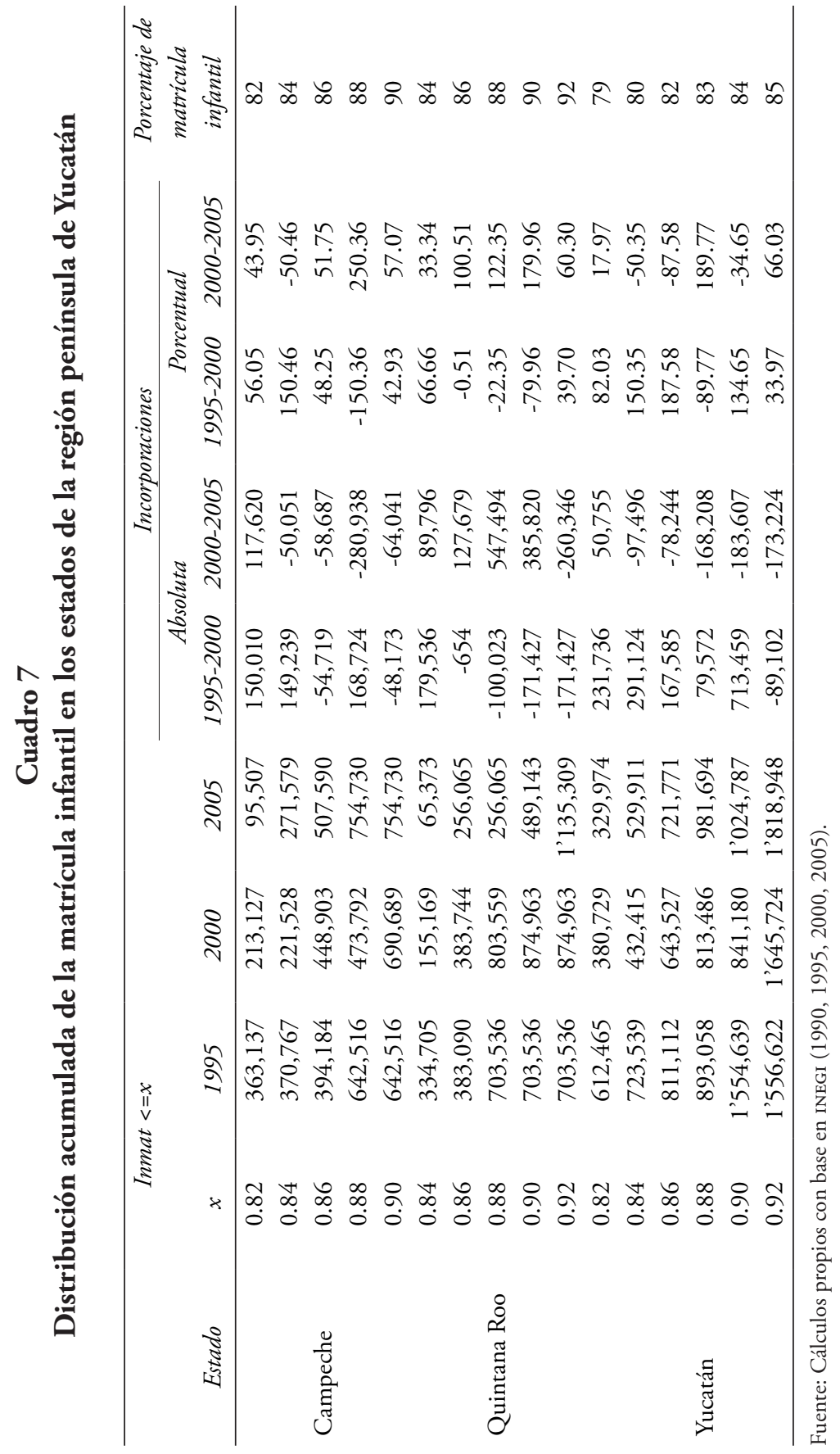




\subsection{PIBP en la región península de Yucatán}

El PNUD (1998: 25) considera el ingreso como el núcleo de una de las tres perspectivas de mayor relevancia en torno a la discusión originada por la definición y medición de la pobreza. Desde esta perspectiva, el ingreso se asocia a la incapacidad monetaria para satisfacer los elementos básicos de la vida, es decir, una persona se considera pobre cuando su nivel de ingreso es inferior a un nivel definido.

En 1995 el pibp en el país fue de 12,415.25 dólares PPA, 1,556.44 menos que en 2005; durante el decenio, México se ubicó en la categoría de Inpibp bajo, según la clasificación del PNUD. El incremento en el decenio fue bajo como consecuencia de un crecimiento importante en el quinquenio 1995-2000 y ninguno entre 2000-2005.

A escala estatal, en los 10 años 13 estados mejoraron su clasificación, donde destaca Michoacán con el mayor avance, al pasar de la posición 29 a la 24, Guanajuato y Zacatecas avanzaron cuatro posiciones, en 2005 se ubicaron en los lugares 18 y 23, respectivamente. Nuevo León fue el único que mejoró en la clasificación y que al principio del periodo se encontraba entre las cinco entidades con mayor PIBP, al pasar del lugar cuatro al dos.

Entre las siete entidades que se mantuvieron en la clasificación estatal se encontró Yucatán en el lugar 19, que en el primer periodo avanzó una posición y la cual perdió en el último quinquenio, a diferencia de las demás entidades de este grupo que no tuvieron cambios. Por otro lado, estos siete estados lograron mejorar su Inpibp en el decenio. En este estrato también se encontraron el Distrito Federal y Chiapas, ocupando las posiciones uno y 31, respectivamente, con PIBP en 2005 de 21,861.61 y 3,886.55 dólares PPA para cada uno.

Campeche, Quintana Roo y otros 10 estados descendieron en la clasificación. Campeche pasó de la posición dos a la tres, a pesar de haber logrado un incremento en su Inpibp de 7.29\%, ya que su PIBP creció 1,841.62 dólares PPA. Quintana Roo, por su parte, pasó del lugar tres al seis, provocado no únicamente por los avances de otras entidades como Nuevo León y Coahuila, que pasó del lugar siete al cuatro, o Chihuahua que pasó del seis al cinco, sino además porque fue el único estado del país donde el Inpibp mostró retrocesos.

Considerando los crecimientos porcentuales del Inpibp, 17 entidades lograron aumentos superiores al nacional, entre los que destacan Nuevo León, Coahuila y Chihuahua, que al inicio del periodo presentaron índices superiores al promedio pero además lograron los máximos incrementos durante el decenio, $14.45,14.37$ y $12.90 \%$, respectivamente. Otros estados que sobresalen por la misma situación son Aguascalientes 


\section{Figura IV}

\section{Inpibp 1995 vs. incremento porcentual Inpibp 1995-2005}

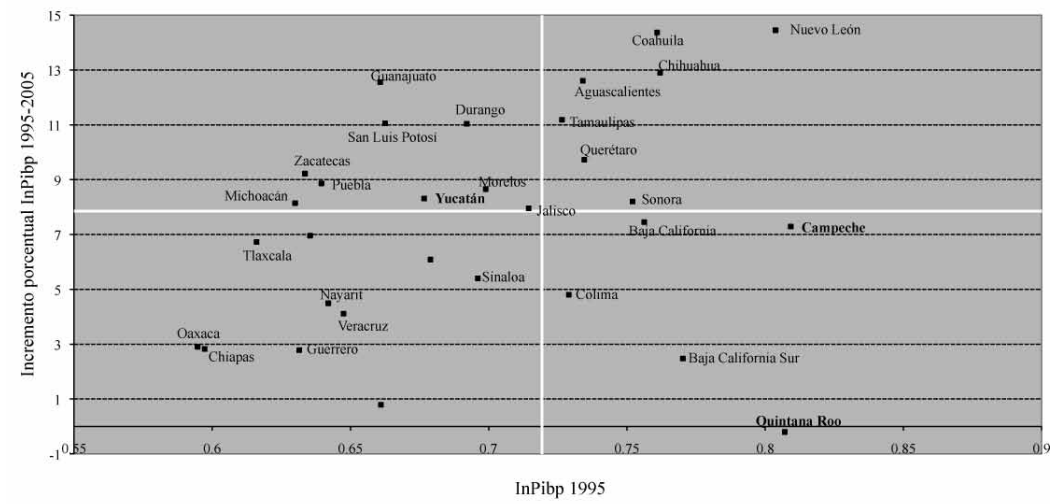

Fuente: Cálculos propios con base en INEGI (1990, 1995, 2000, 2005).

(12.60), Distrito Federal (12.45), que en el primer quinquenio logró el incremento más alto y en el segundo la peor pérdida, Tamaulipas (11.18), Querétaro (9.37) y Sonora (8.20).

En el otro extremo se encontraron los estados de Tabasco (0.29), Guerrero (2.78), Chiapas (2.83), Oaxaca (2.90), Veracruz (4.11) y Nayarit (4.49), entres otros, como los más desprotegidos económicamente, ya que a pesar de encontrarse por debajo del Inpibp nacional no lograron superar el incremento porcentual del país en el decenio.

$\mathrm{El}$ ingreso, medido con el PIBP, muestra un comportamiento mucho más irregular que los otros componentes del DH, se aprecia una desaceleración drástica en el segundo quinquenio, ya que los crecimientos presentados durante los primeros cinco años fueron ampliamente superiores en la mayoría de los estados. En el primer quinquenio ninguna entidad mostró decrementos en el PIBP, mientras que para el segundo, 15 los presentaron, el mismo país cayó $0.35 \%$ en su Inpibp.

Una gran cantidad de estados no se ajustan a la tendencia esperada, lo cual sí ocurre con las entidades de la península. Se tenía una polarización más o menos clara, donde los estados con las mejores condiciones económicas se encuentran más cercanos al norte, y por otro lado, a excepción de Nayarit y Sinaloa, las entidades más desprotegidas económicamente como Guerrero, Chiapas, Oaxaca, Veracruz y Tlaxcala se encuentran cercanos unos de otros en el sur del país.

En lo que respecta a la contribución de la región al PIB nacional, en 2005 fue de 4.12\%, equivalente a 64’713,021,000 de pesos en términos corrientes. De los estados de la península que más aportó al PIB regional fue Quintana Roo: con 37.19\%, superando a Campeche, donde la pro- 
ducción petrolera permitió que contribuyera con $28.77 \%$ a pesar de ser el estado menos poblado de la península; Yucatán aportó 34.04\%. En el estado de Campeche la actividad que más aporta al PIB estatal es la minería (extracción de petróleo) con más de 53\%, seguida por los servicios (19.2) y las actividades comerciales (7.8); en 1995 la población económicamente activa (PEA) era de 183,679 personas, $28.59 \%$ de la población estatal. En 2000 y 2005 la PEA llegó a representar 35.56\% (245,660 habitantes) y 43.16\% (325,715 habitantes), respectivamente, de la población estatal. En Yucatán son importantes las industrias alimentaria, del vestido y calzado, de la construcción, la mueblera, salinera, pesquera y la fruticultura, sobre todo cítricos. En el año 1995 la PEA era de 473,710 habitantes, equivalente a $30.43 \%$ de la población estatal y, conforme la población del estado aumenta su edad promedio, crece la PEA. En el 2000 representaba $37.57 \%$ (623,033 habitantes) y en $2005,43.05 \%$ (783,118 habitantes). En 1995 la PEA en Quintana Roo era de 235,176 personas (33.43\% de la población del estado); al igual que en los otros dos estados la dinámica poblacional trajo consigo un incremento en la PEA, ya que para 2005 llegó a 47.44\% de la población, equivalente a 538,317 habitantes. En Quintana Roo las principales actividades económicas son las relacionadas con el turismo, como el comercio, los restaurantes y hoteles que en 2004 ocupaban más de 50\% de la PEA estatal. Parte el aumento en la edad promedio de los habitantes y parte el actual esquema económico han traído un cambio sustantivo en la PEA de los estados del país, como se muestra en los de la región península.

En el cuadro 8 se muestra que en Campeche ninguna proporción de la población en 1995 tenía Inpibp entre 0.82 y 0.90, y entre 0.82 y 0.94 para 2000 y 2005, debido a la buena condición económica prevaleciente en el municipio del Carmen, principal productor de petróleo del país, que al inicio del periodo tenía un Inpibp de 0.90908 y en 2005 de 0.94955 , el segundo más alto del país. Un fenómeno similar ocurrió en Quintana Roo, donde la buena situación de los municipios de Cozumel e Isla Mujeres en 1995, con Inpibp de 0.90523 y 0.94453 , respectivamente, superaban a Benito Juárez, principal destino turístico en México. A pesar de las ventajas económicas el estado tuvo un retroceso, debido en gran medida a los huracanes ocurridos durante el decenio. Yucatán evidenció la peor situación económica de la región, ya que su rango de ingresos está desfasado hacia los ingresos más bajos y en 2005, 93 de los 106 municipios yucatecos se encontraban por debajo de un Inpibp de 0.70; existe una gran concentración de población en Mérida, la cual en 2005 tenía más de $40 \%$ de la población estatal, razón por la que se observa gran cantidad de personas en la categoría más alta alcanzada por el estado. 


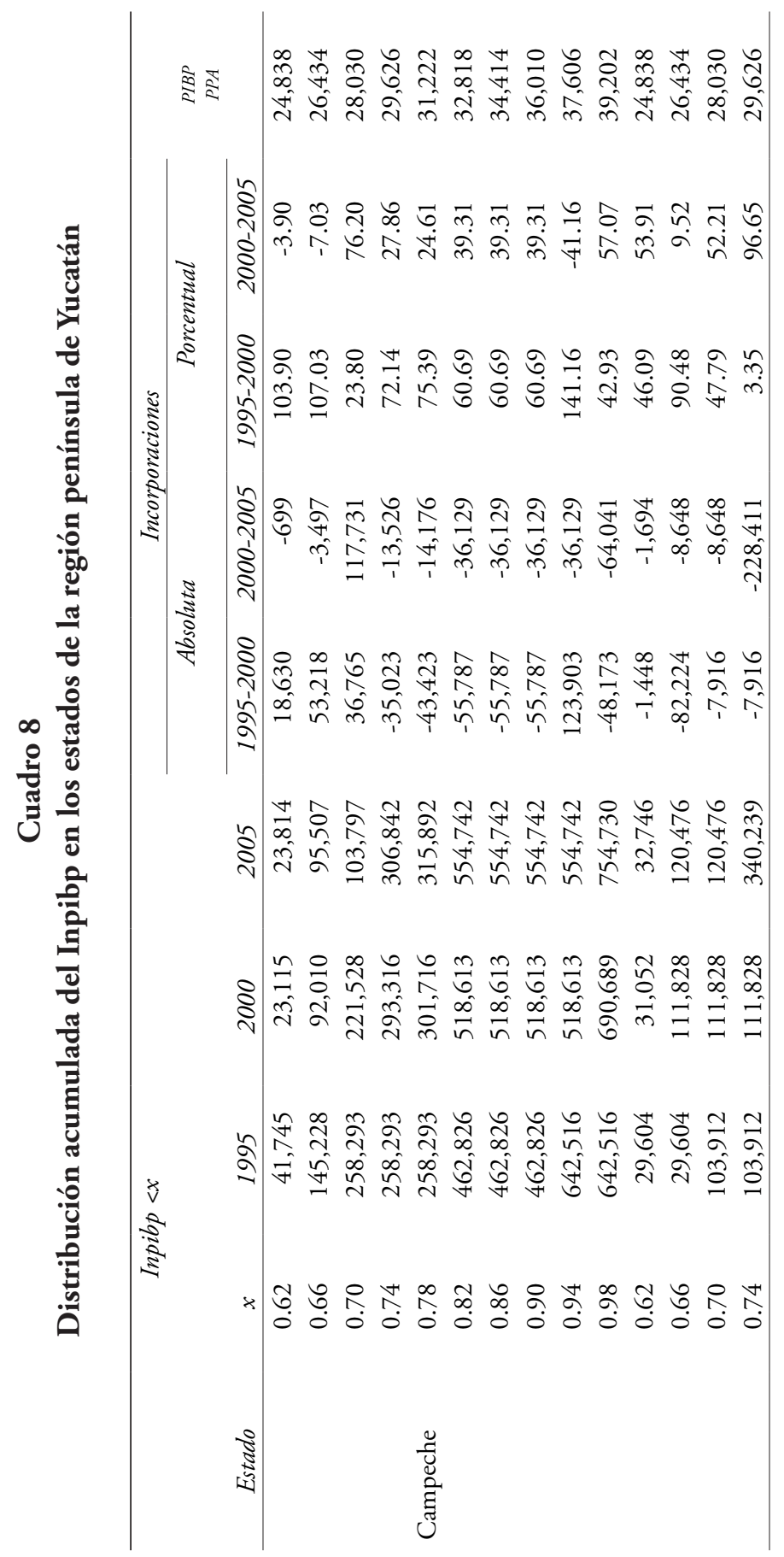




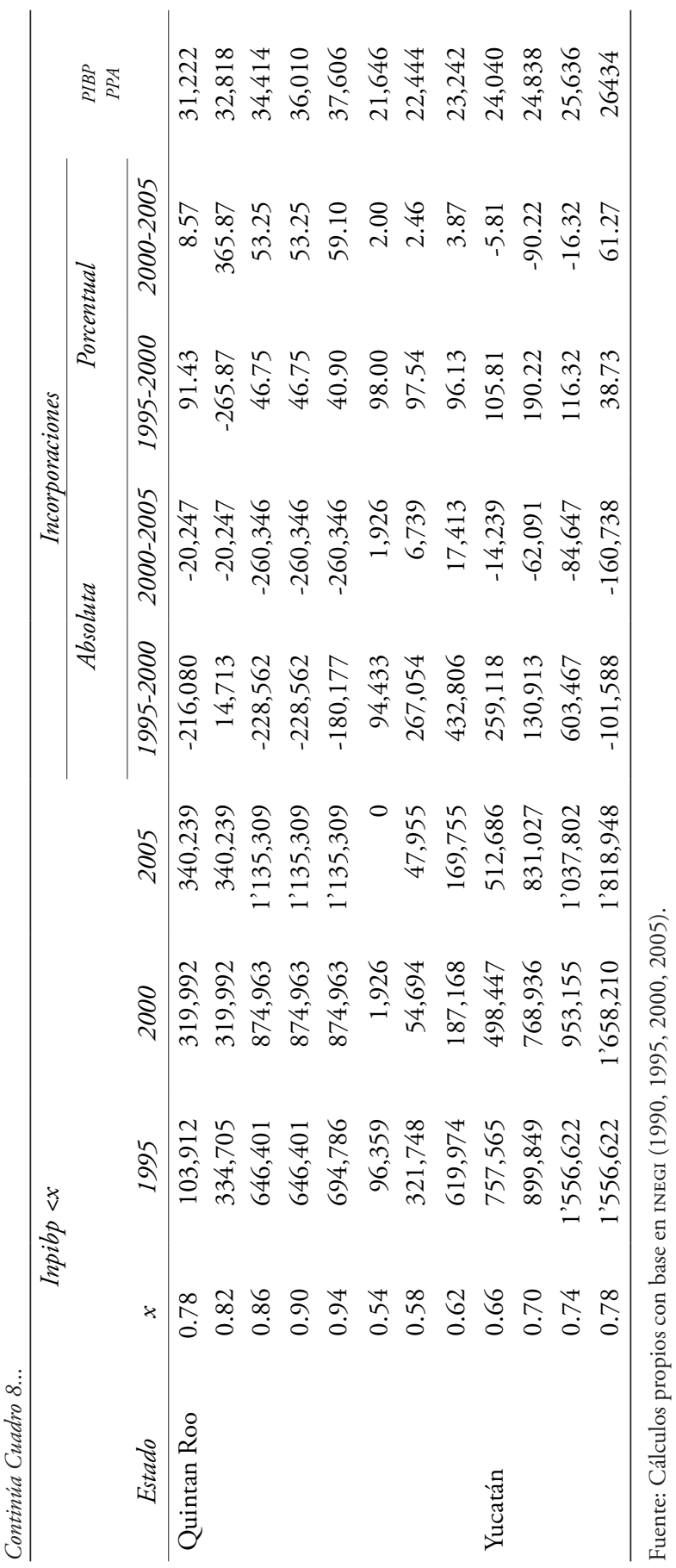




\subsection{IDHP en la región península de Yucatán}

En las secciones anteriores se analizó la situación y cambios de los componentes del IDHP para los estados de la península de Yucatán en el contexto nacional. En esta sección se estudia el IDHP que recoge la expresión de todos ellos en una medición del DH.

Fueron 13 las entidades que lograron mejorar su clasificación en el IDHP, siendo Guanajuato el que tuvo la mayor ganancia de posiciones, pues pasó del lugar 24 al 21, cambio logrado por el avance que tuvo en el segundo quinquenio del periodo, sin embargo, en 2005 aún no lograba un IDHP alto de acuerdo con la clasificación del PNUD. Coahuila, Aguascalientes, Chihuahua y Tamaulipas mejoraron en la clasificación, manteniéndose entre las 10 mejores posiciones de DH del país. En el año 2000 Yucatán pasó del lugar 22 al 20, y se posicionó al final del decenio en el lugar 19.

Entre los 11 estados que mantuvieron su posición en la clasificación se encontraban el Distrito Federal y Nuevo León, que estaban en los lugares primero y segundo, respectivamente; en contraste, también en este grupo se encontraron Chiapas y Oaxaca en los últimos lugares con las posiciones 32 y 31 , respectivamente; lo cual refleja las muy buenas o malas condiciones que persisten en estas entidades, según sea el caso, en los tres componentes del IDHP, que como se ha observado en las secciones anteriores, esos estados siguen una dinámica de DH diferente a la del resto del país.

Baja California Sur tuvo la peor pérdida de posiciones al pasar de la cuatro a la ocho, junto a Quintana Roo que pasó de la tres a la cuatro, fueron los únicos de este estrato que en el inicio del periodo tenían un IDHP alto y en 2005 se encontraban entre los 10 primeros; además fueron los únicos del país que en el segundo quinquenio retrocedieron en el valor del índice, razón por la que fueron desplazados en la clasificación por otras entidades que lograron incrementos importantes. El caso de Quintana Roo se explica por los desastres naturales, no así el de Baja California Sur.

Todas las entidades del país incrementaron su IDHP durante el decenio, 19 crecieron menos que la media nacional (8.12\%), destacando los casos de Chiapas, Oaxaca, Estado de México, Guerrero, Zacatecas, Veracruz, Tabasco y Nayarit, que en 1995 tenían un IDHP por abajo del nacional (0.77023), es decir, las políticas públicas y medidas tomadas por los gobiernos locales no fueron efectivas ni suficientes para reducir la brecha entre las opciones de desarrollo que tienen estas localidades y las del resto del país. 
Por otro lado, lo estados de Querétaro, Coahuila, Tamaulipas, Aguascalientes, Campeche y Colima, que en 1995 tenían un IDHp superior al del país, durante el decenio lograron incrementos del índice mayores al crecimiento promedio nacional.

El resto de las entidades se ajustaron a lo esperado, es decir, que los que comenzaron el decenio en una mejor situación presentaron menores incrementos de DH. Quintana Roo a pesar de que tuvo el menor crecimiento porcentual en la región es el que ha mostrado los mejores indicadores de desarrollo, a diferencia de Yucatán donde sus elevados incrementos se deben a lo antes mencionado.

En la figura $\mathrm{v}$ se observa que aquellos estados menos desarrollados $\mathrm{y}$ con menor bienestar para su población, como Chiapas, Oaxaca, Guerrero, Veracruz, Tabasco, e incluso Nayarit, siguen una tendencia bien definida al igual que el resto de las entidades, es decir, los menores incrementos los tienen los estados que al inicio del periodo tenían los IDHP más altos, sin embargo, siguen la misma dinámica pero en niveles inferiores al resto. Inclusive se podría determinar otro estrato superior al anterior, conformado por estados como Puebla, Michoacán, Tlaxcala, el Estado de México y las Baja California, además de un grupo superior a éste, compuesto por Hidalgo, Yucatán, Morelos, Jalisco, Sinaloa y Quintana Roo. Cada grupo, a pesar de las diferencias en los índices y sus incrementos, tendría en común la eficiencia de las políticas públicas y sociales encaminadas a mejorar el DH dadas las condiciones iniciales de las poblaciones. En estos grupos existiría la posibilidad de comparar los cambios en la medición del desarrollo en términos absolutos y no relativos. Finalmente se muestran los estados de Aguascalientes, Campeche, Coahuila y Tamaulipas, donde las acciones realizadas por los diversos sectores sociales y políticos permitieron una aceleración en el desarrollo, comparable a la de Nuevo León y el Distrito Federal.

En 2005, 17 localidades superaron el IDHP de 0.8, el único estado de la península que no lo logró fue Yucatán, es decir, Campeche y Quintana Roo contaban con las condiciones mínimas necesarias para que su población tuviese acceso a servicios de salud, educación y un ingreso que les permita vivir dignamente.

En la figura vi se aprecia claramente que no existieron decrementos en la medición del DH para ninguna entidad. La ganancia o pérdida de posiciones de los estados en la clasificación del IDHP se da como resultado de la magnitud de los avances alcanzados por cada estado en $\mathrm{DH}$, una caída en la clasificación no indica un retroceso en el desarrollo, sino un menor crecimiento de la entidad, razón por la cual se ve superada por otras que al inicio del periodo mostraban condiciones ligeramente inferiores. Por ejemplo, Quintana Roo cae una posición ya que Coahuila, que 


\section{Figura V}

IDHP $1995 v s$. incremento porcentual IDHP 1995-2005

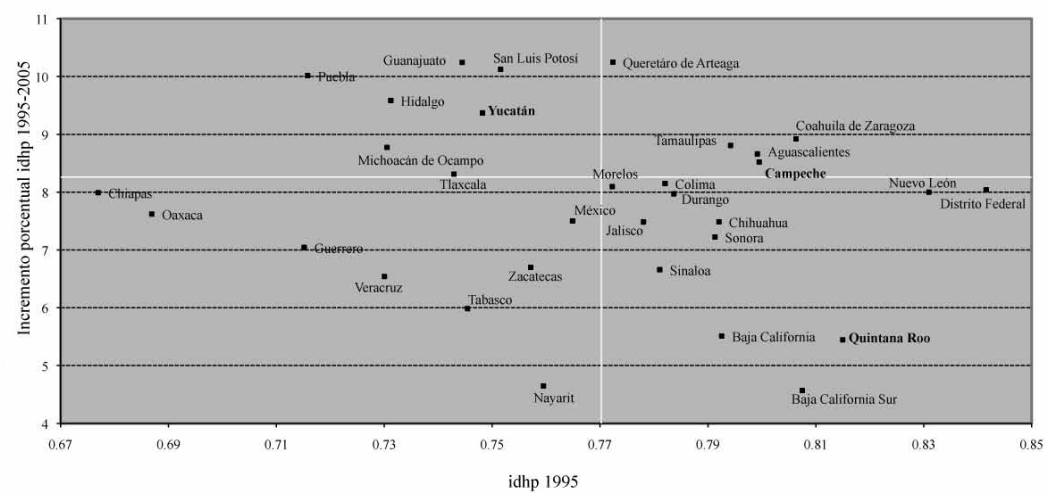

Fuente: Cálculos propios con base en INEGI (1990, 1995, 2000, 2005).

\section{Figura VI}

Avances en posición $v s$. incremento porcentual, IDHP, 1995-2005

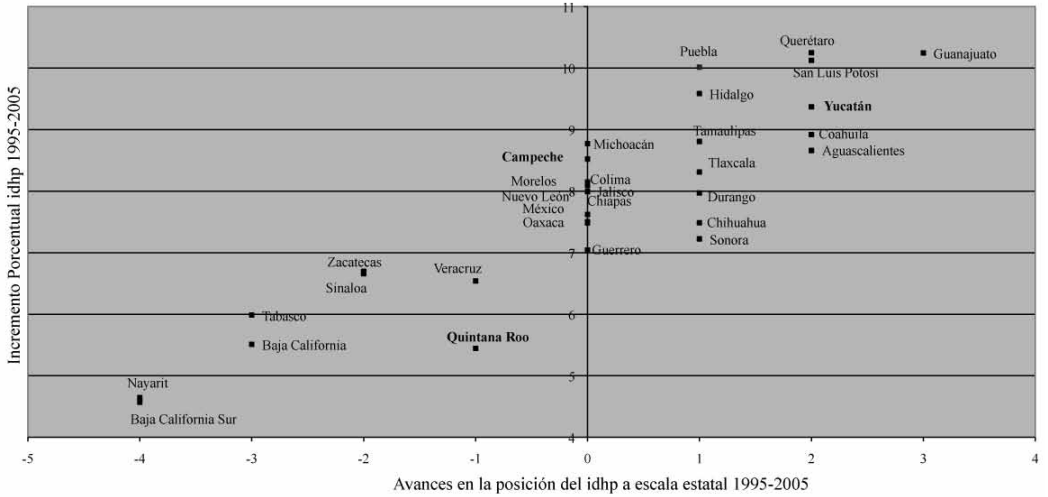

Fuente: Cálculos propios con base en INEGI (1990, 1995, 2000, 2005).

tenía un IDHP menor, pero muy cercano al del estado de la península, tuvo un crecimiento muy superior.

Se espera que, a través del tiempo, las poblaciones de los diferentes estados avancen en el desarrollo, es decir, que cada vez más personas se incorporen a categorías más altas de IDHP y de sus componentes, abandonando de manera paulatina en su totalidad las categorías inferiores. La creación durante el primer quinquenio de los municipios de Calakmul y Candelaria que se escindieron de los municipios de Campeche y el Carmen, respectivamente, ocasionó el aumento del número de personas con IDHP menor a 0.78 en el periodo. Además, se aprecia que las localidades con menor bienestar en la región se localizan en el estado de Yucatán. Duran- 


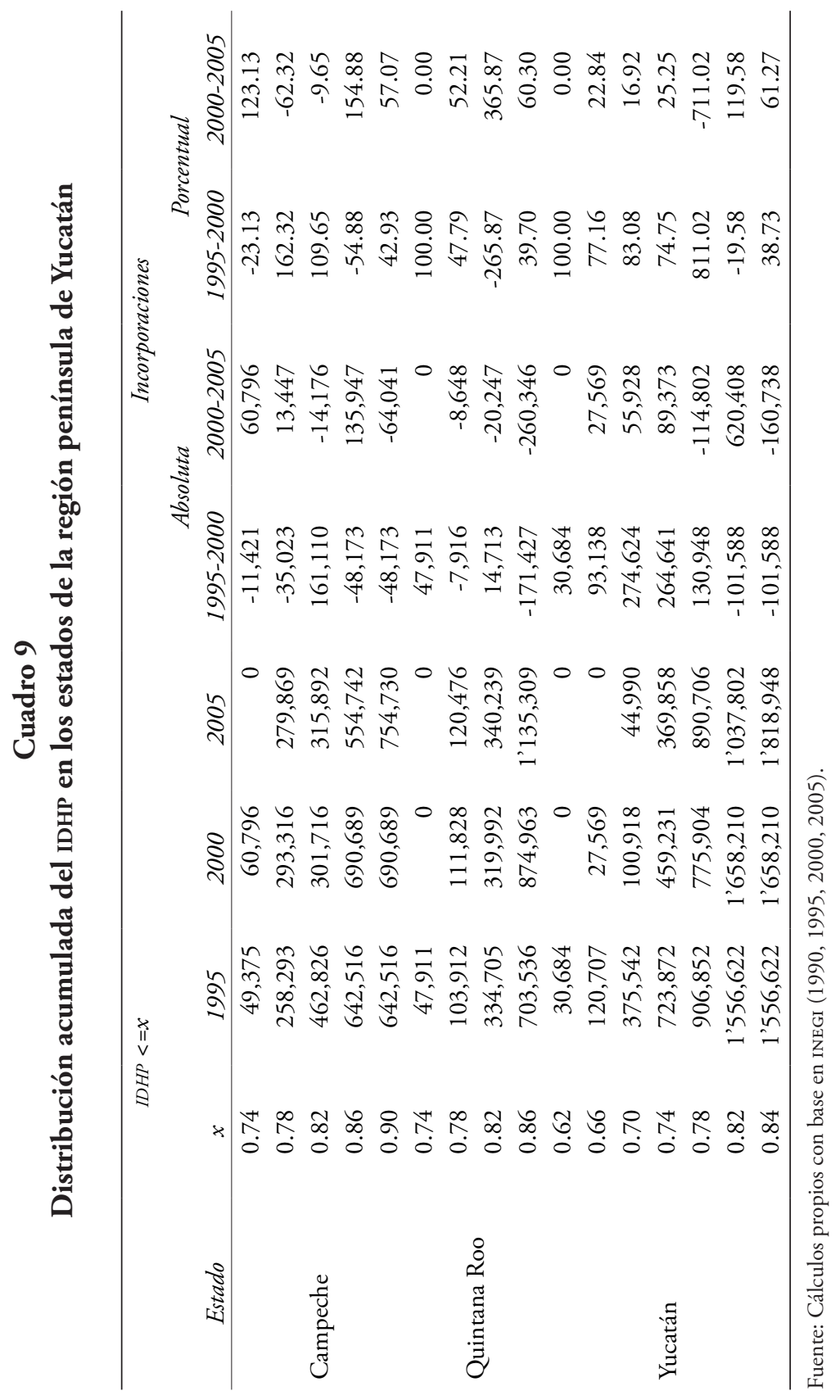




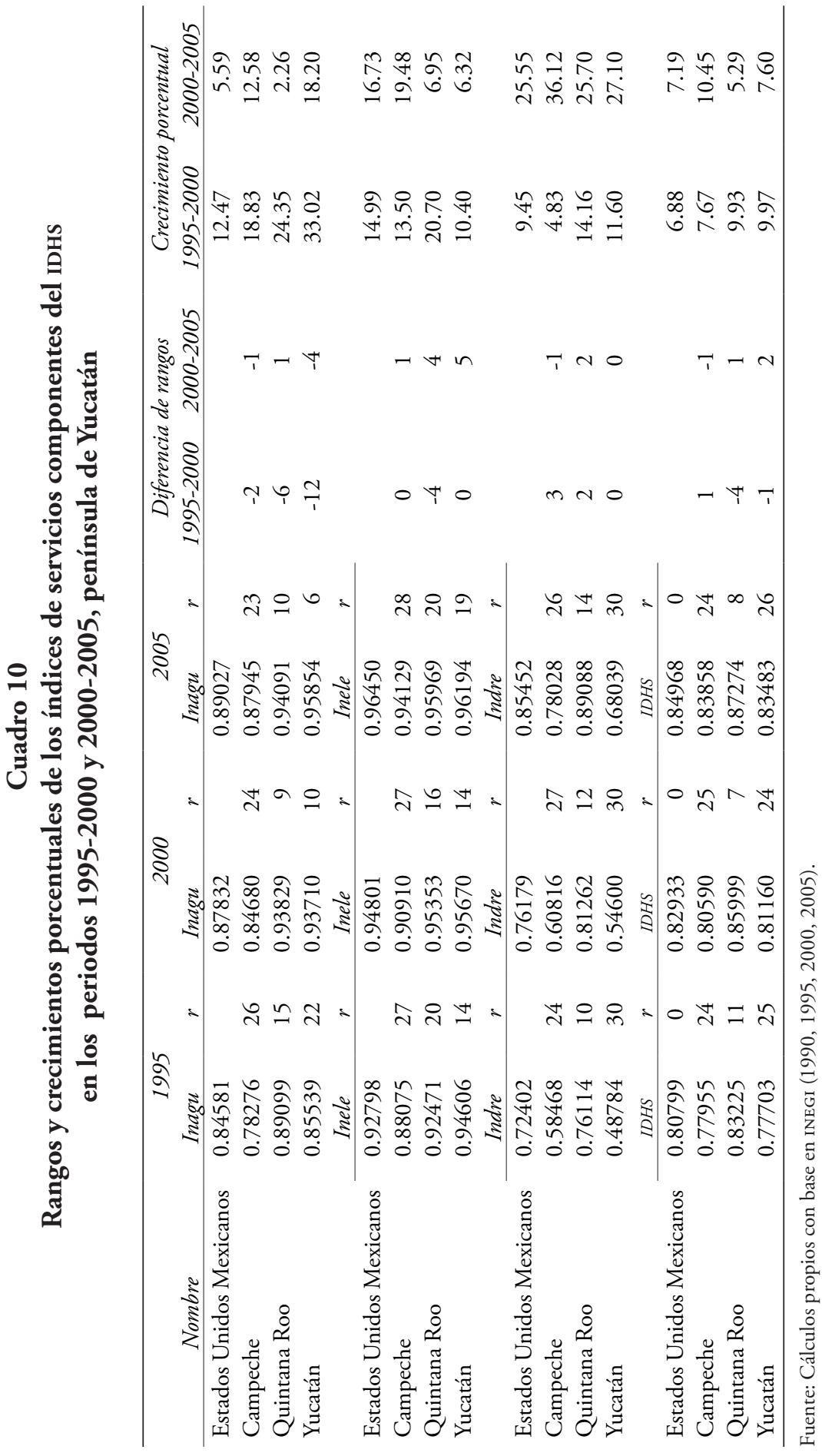


te el segundo quinquenio el aumento de personas en las categorías de IDHP menor a 0.82 en Quintana Roo, muestra el efecto que entre otros factores tuvieron los desastres naturales sufridos por dicho estado (cuadro 9).

\subsection{Servicios (agua, electricidad y drenaje) en la región península de Yucatán}

El abastecimiento de agua potable, drenaje y electricidad en condiciones de calidad y cobertura adecuada son de gran importancia para elevar los niveles de bienestar social en la población. En México la población crece a una tasa cercana a 1\% anual (INEGI, 2010), por lo que representa un reto mantener e incrementar la cobertura de los servicios básicos para reducir el rezago existente.

La cobertura de estos servicios en todas las zonas del país es resultado de las funciones de diferentes instituciones de los gobiernos federal, estatal y municipal, y de una afluencia de empresas privadas con una participación financiera cada vez más importante. La población usuaria forma parte importante del proceso de expansión y mejora de los servicios, por la contribución que realiza con el pago del servicio y por el cuidado y mantenimiento de la infraestructura y recursos que recibe.

En 2005 México tenía 103'263,388 habitantes, de los cuales 89\% contaba con agua entubada, $96.5 \%$ con energía eléctrica y $85.5 \%$ con el servicio de drenaje. De 1995 a 2005 la población del país aumentó en $12 ’ 105,098$ personas. Al inicio del decenio, del total poblacional, 84.6\% disponía de agua entubada de la red pública, $92.8 \%$ de energía eléctrica y $72.4 \%$ de drenaje. La expansión de la cobertura de cada uno de los tres servicios se ha dado a una mayor velocidad que el crecimiento de la población, donde el drenaje es el de mayor cambio, reduciendo el rezago ancestral que muestra respecto a los otros dos servicios. Respecto al servicio de agua potable, 13 entidades mejoraron en la clasificación a escala estatal, Yucatán obtuvo el máximo avance al pasar de la posición 22 a la seis, debido a que presentó el mayor incremento porcentual del Inagu en el periodo. Quintana Roo también mejoró su posición y presentó un considerable aumento en la cobertura del servicio, a pesar de que en el decenio su población aumentó en 431,773 habitantes, 61\% de la población estatal en 1995; mientras que Campeche logró ascender en la clasificación pero sin poder superar la cobertura promedio del país.

De los otros 19 estados, tres mantuvieron su posición, aquí resalta Aguascalientes que ocupó el primer lugar durante los años de análisis, a pesar de ser uno de los dos estados que mostraron pérdidas en la cobertura del servicio, ya que la población beneficiada disminuyó 6\%, quizá porque la población total creció más de $20 \%$. Entre las 16 entidades que 
empeoraron, Chiapas, Oaxaca y Guerrero siguen mostrando grandes atrasos y una dinámica similar pero con menor ritmo que los demás estados; en esas tres entidades aproximadamente $70 \%$ de sus habitantes contaba con agua potable.

Los estados de la península de Yucatán, y otras 16 entidades, lograron incrementar el Inagu en un porcentaje mayor al nacional, de ellos Campeche y otros siete tenían un valor del indicador menor al del país en 1995. De las entidades que crecieron menos que el promedio nacional, sólo Oaxaca y Guerrero no tenían una cobertura del servicio de agua potable superior a la media nacional. Es decir, la mala situación de estados como Tabasco, Veracruz, Chiapas, Oaxaca y Guerrero provocó que el Inagu nacional no superara el 0.90 en 2005.

El servicio de energía eléctrica es el que presenta las mejores condiciones a lo largo y ancho de la República. En 2005 la cobertura mínima del servicio la presentó Oaxaca con $92 \%$, mientras que para el mismo año el Distrito Federal logró la máxima cobertura: 98.5\%. Existen amplias diferencias entre estos dos estados, una razón en particular es la proporción de población que habita en zonas rurales en cada uno de ellos.

Entre las 15 entidades que mejoraron su clasificación en el Inele a escala estatal, no se encontraban los estados de la península de Yucatán. Zacatecas presentó el mayor avance al pasar de la posición 18 a la 10, gracias a un incremento en el índice de $33.98 \%$, el quinto más alto en el decenio. Otro de los 15 estados que llama la atención es Chiapas, uno de los principales generadores de energía eléctrica en el país. Chiapas pasó de la posición 32 a la 30, pero logró el mayor incremento estatal del Inele, $45.16 \%$, pasó de 0.77166 a 0.93080 , sin duda un avance sustancial en la reducción de su rezago.

Quintana Roo, Sonora, Jalisco y el Distrito Federal mantuvieron sus posiciones, 20, 13, nueve y uno, respectivamente. Los tres primeros perdieron en el segundo quinquenio el mismo número de posiciones ganadas en el primero y el Distrito Federal se mantuvo en el primer lugar desde 1995. Quintana Roo es el único de los cuatro que al inicio del decenio se encontraba por debajo de la cobertura promedio en México, situación que pudo revertir en el año 2000, viéndose superado por la media nacional nuevamente en 2005. Además sólo el Distrito Federal presentó decrementos en el indicador al pasar de 0.99839 a 0.98531 , pues mientras su población aumentó en 231,909 personas, el número de beneficiarios creció en 117,466.

Campeche, Yucatán y otros 11 estados cayeron en la clasificación. Campeche fue del lugar 27 al 28, con el undécimo incremento más grande del Inele, viéndose rebasado por Veracruz que tuvo un crecimiento de 41.21\%. Yucatán perdió cinco posiciones, cayó al lugar 19 al tener uno 
de los siete incrementos más bajos del índice, lo cual motivó que lo superaran estados con crecimientos porcentuales más grandes como Tabasco, Puebla y Michoacán.

De las 12 entidades, entre ellas Campeche, que tuvieron aumentos porcentuales del Inele superiores al del país durante el decenio, únicamente Michoacán se encontraba por arriba del Inele nacional en 1995. Sólo Zacatecas accedió al grupo de los 10 estados con mayor cobertura del servicio al final del periodo. Campeche fue el único que retrocedió en la clasificación al caer una posición.

De los 20 estados restantes, Guerrero, Quintana Roo, Oaxaca y Chihuahua se encontraban por debajo de la media en 1995, se les unió en 2005 Baja California Sur, Baja California, Yucatán y Nayarit. El Distrito Federal y Morelos fueron los únicos que en 2005 presentaron pérdidas porcentuales del índice, razón por la que Morelos cayó de la posición dos a la cinco.

Respecto al Indre, Nayarit y Zacatecas avanzaron cuatro posiciones, los mayores logros en la escala estatal, como resultado de ampliaciones del índice de 42.04 y $53.55 \%$, respectivamente. Tabasco y Nayarit en 1995 se ubicaban entre los 10 Indre más altos, Nayarit y Colima se incorporaron a este grupo en 2005.

Entre los 10 estados que mantuvieron su posición se encontraban: el Distrito Federal, Colima, Aguascalientes, Jalisco y Nuevo León, que ocupaban las primeras cinco posiciones en el orden mencionado, además eran los únicos del grupo que en 1995 tenían cobertura del servicio de drenaje superior a la nacional. También en este grupo se encontró Yucatán que se ubicó en el lugar 30.

De las entidades que retrocedieron, Campeche fue de la posición 24 a la 26 y Quintana Roo pasó de la 10 a la 14, a pesar de haber tenido incrementos considerables en la cobertura del servicio. De este conjunto, en 2005, el Estado de México mostró las mejores condiciones al ubicarse en la posición nueve.

El Indre de 25 estados, incluidos Campeche, Quintana Roo y Yucatán, creció más que el nacional en el decenio, sin embargo, en 1995 sólo nueve estados se ubicaban con respecto a este servicio por encima de la cobertura media nacional. En este estrato se encontraron los 12 estados con menor cobertura durante el decenio de análisis. Quintana Roo fue el único estado de la Península que en el inicio del periodo tenía un Indre superior al del país.

Considerando los valores de los tres índices de servicios para los 125 municipios que componían la península de Yucatán en 2005, se obtuvieron correlaciones significativas al 5\% entre el Inele y el Indre (0.3505), entre el Inele y el Inagu (0.2755), razón por la cual el análisis de incor- 
poración se llevará a cabo empleando el Inserv, que es la media aritmética de los tres índices de servicios, como un indicador de las condiciones de servicios generales predominantes en la región.

Para los tres estados de la península, el amplio rango del Inserv que se observa en el cuadro 11 se debe a las deficientes condiciones de los tres servicios en comunidades poco pobladas; se aprecia poca concentración de población en las categorías más bajas y un aumento considerable en la categoría que va de 0.94 a 0.98 , donde se localizan los municipios de mayor tamańo, como la capital del estado de Campeche, Solidaridad y Benito Juárez en Quintana Roo, y Mérida y Progreso en Yucatán.

El servicio de drenaje es el que ha mostrado la menor cobertura en el territorio nacional, aunque se logró un salto en los años posteriores al 2000. Dicha escasez del servicio no ha tenido una distribución territorial regular, la península de Yucatán es una de las regiones con mayores problemas en cuanto a drenaje, esto debido al tipo de suelo predominante en la región que no permite la excavación para disminuir el número de viviendas que no poseen el servicio. Al igual que en la mayoría del país, el servicio de energía eléctrica fue el que tuvo la mayor cobertura en la región. Campeche cuenta con tres centrales generadoras de electricidad de diferente tipo, en Quintana Roo operan seis y en Yucatán se tienen ocho (Castro, 2007).

\subsection{IDHS en la región península de Yucatán}

En esta sección se analiza el IDHs que es una herramienta alternativa al IDHP para medir el DH, sustituyendo la aportación que tiene el PIBP al desarrollo, por la contribución que trae consigo el contar con tres servicios básicos (agua, drenaje y electricidad).

En la clasificación del IDHs, 10 estados mejoraron su posición. Los mayores avances correspondieron a Sinaloa y Tamaulipas con cuatro posiciones, ubicándose en 2005 en los lugares nueve y 12, respectivamente. Otros estados que destacaron son Aguascalientes y Nuevo León que, al crecer más de $5.26 \%$ con respecto al Distrito Federal, se ubicaron en las posiciones uno y dos, respectivamente. Quintana Roo pasó del lugar 11 al ocho, desplazando a estados como Morelos y Baja California Sur.

Entre las nueve entidades que mantuvieron su posición se encontraron Colima y Coahuila, como cuatro y cinco, respectivamente. Guerrero se mantuvo en la posición 30, a pesar de haber tenido un aumento del IDHS de $13.55 \%$, superior al nacional. Campeche no abandonó durante el decenio la posición 24 , su IDHS mejoró $17.17 \%$, sin embargo, durante todo el decenio se localizó por debajo de la media nacional con respecto a esta medición. 


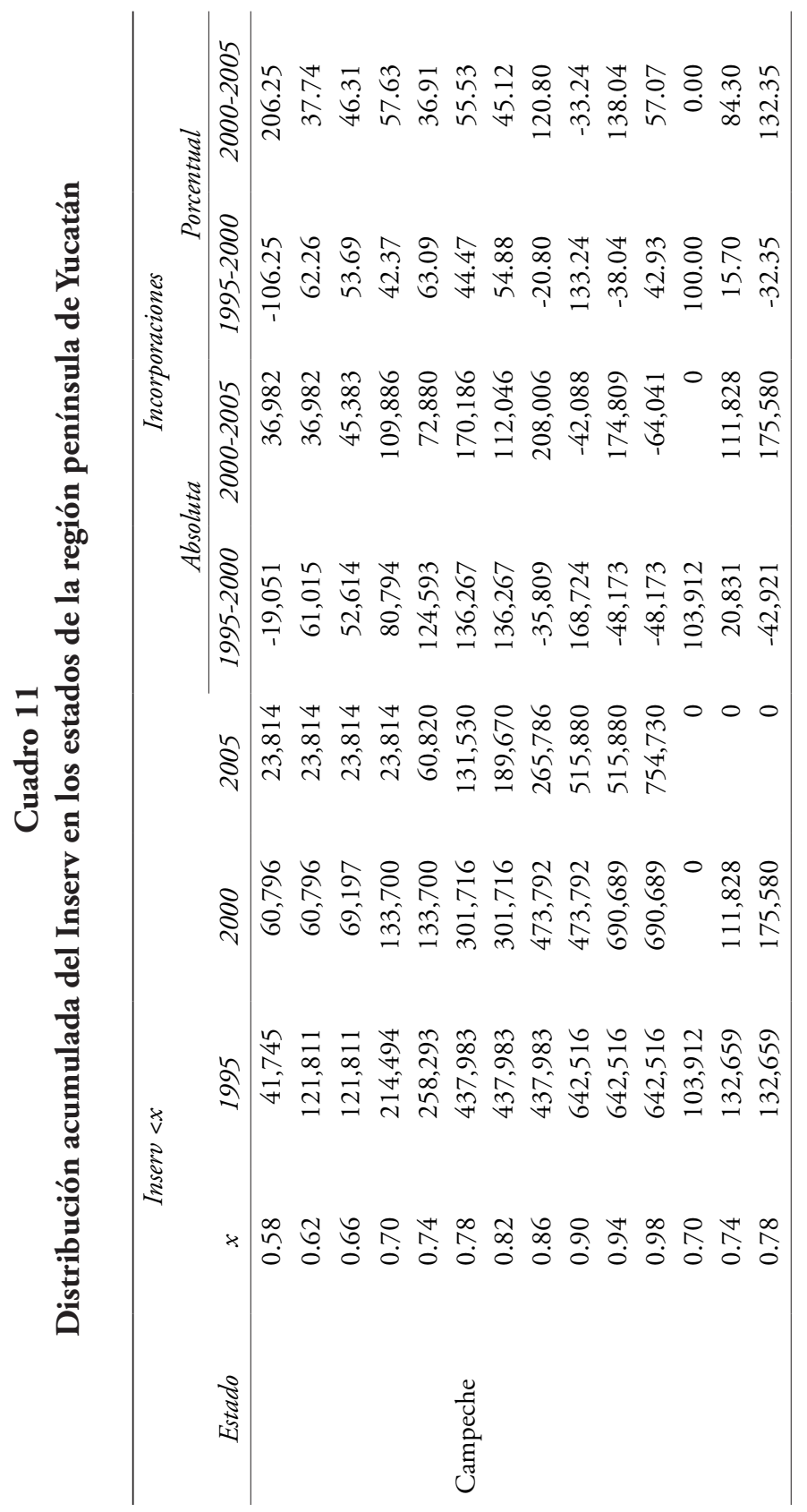




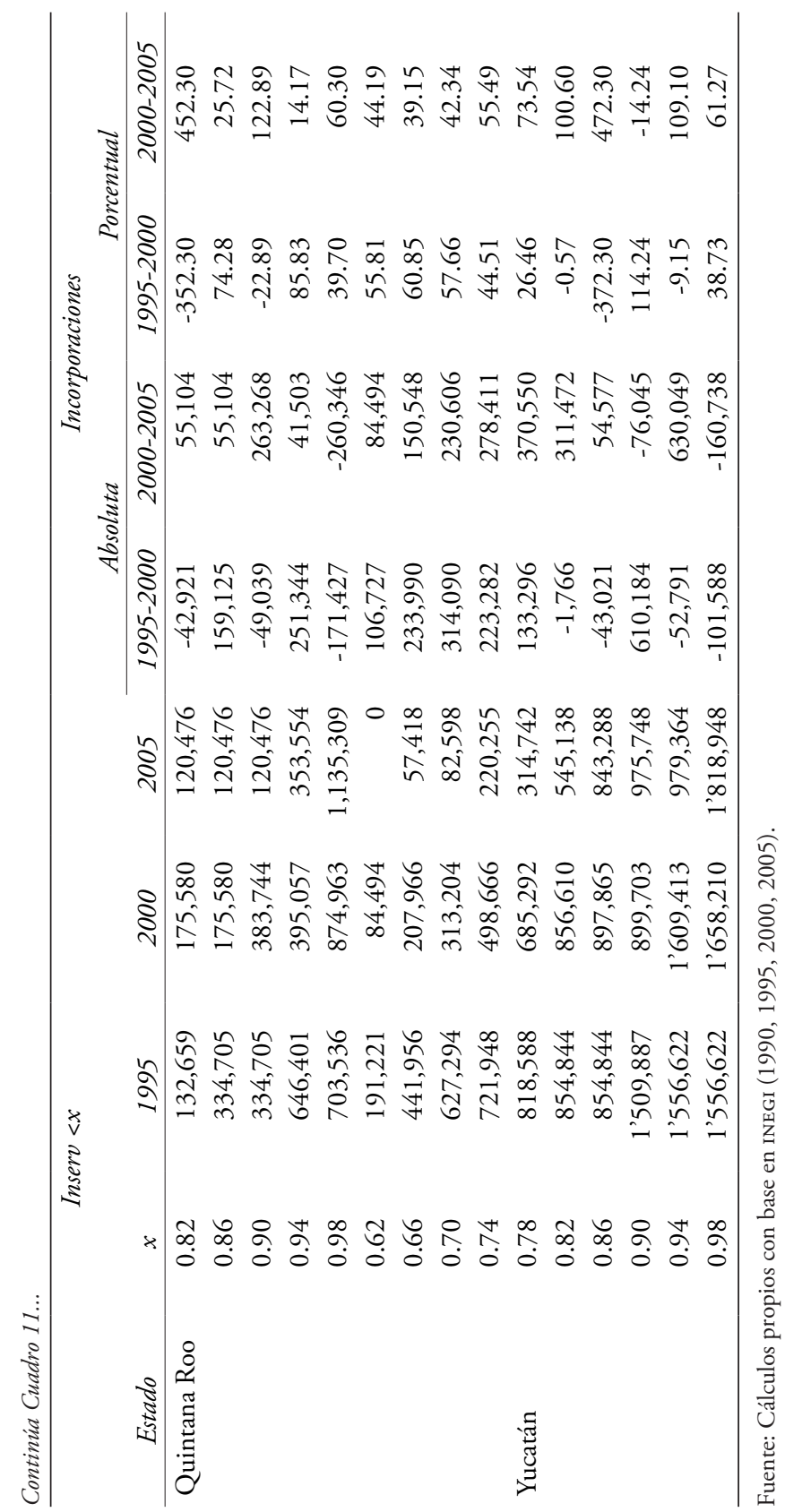


Chihuahua retrocedió más que cualquier otro estado al ir de la posición 14 a la 20, fue uno de los seis estados con crecimientos del índice menores a $10 \%$. El Distrito Federal perdió dos posiciones: cayó al lugar tres. Yucatán pasó de la posición 25 a la 26, a pesar de haber logrado acrecentar su IDHS en $16.68 \%$. Oaxaca cayó hasta la posición 32, persistiendo también una mala situación en el acceso a los servicios de la entidad.

Oaxaca, Guerrero y Chiapas fueron los estados que en 2005 no habían logrado sobrepasar el IDHs de 0.8 , es decir, considerando los criterios del PNUD, estas entidades no contaban con la infraestructura y equipo mínimos necesarios para que su población estuviera en posibilidad de tener acceso a los servicios de salud, educación y contar con agua, drenaje y energía eléctrica. Cabe destacar que son las tres entidades con menor PIвP.

Campeche fue el único estado que en 1995 y 2000 tuvo mayor IDHP que IDHs, lo cual se debe a que la mayoría del PIBp estatal proviene de la producción de petróleo, sin embargo, se desconoce qué proporción de los ingresos generados por esa actividad se quedan en el estado y contribuyen en la generación de condiciones e infraestructura que propicien la posibilidad de un mejor nivel de vida para los campechanos. En la figura VII se muestra que, en general, el IDHs es mayor que el IDHP; los dos puntos que se encuentran por debajo de la recta de pendiente uno son los correspondientes a Campeche para los años 2000 y 2005.

Fueron 18 los estados que en el decenio tuvieron incrementos porcentuales del IDHs mayores al del país (13.44\%). De ellos, Tamaulipas, Sinaloa, Quintana Roo y Tlaxcala en 1995 tenían un IDHs superior al nacional. Yucatán y Campeche, con aumentos de 16.68 y 17.17\% durante el decenio no pudieron superar la media nacional (cuadro 10).

La figura viII revela que al sustituir el рIBP por los servicios mencionados, en la medición del desarrollo, los estados de Guerrero, Oaxaca y Chiapas siguen reflejando cierto retroceso, sin embargo, comienzan a integrarse a la dinámica del resto de los estados. En el caso del IDHS, así como el de los índices expuestos anteriormente, lo ideal sería que la tendencia convergiera de manera paulatina en una línea recta casi perfecta, sin embargo esto resulta complicado dadas las diferentes condiciones culturales, políticas y sociales persistentes en cada estado, inclusive muchas veces mayores dentro de ellos.

En los tres estados, los municipios que los conforman están en un proceso constante de mejora en cuanto al $\mathrm{DH}$, cada vez más personas se incorporan a categorías más altas del IDHS, y el mínimo índice municipal para cada entidad está en aumento. En el cuadro 12 Campeche muestra una considerable parte de población en la categoría más alta para cada uno de los años, la razón al igual que con el IDHP, es que los dos municipios más poblados en el estado, Carmen y Campeche, son los que han 
Figura VII

IDHP estatal $v s$. IDHS estatal, 1995, 2000, 2005

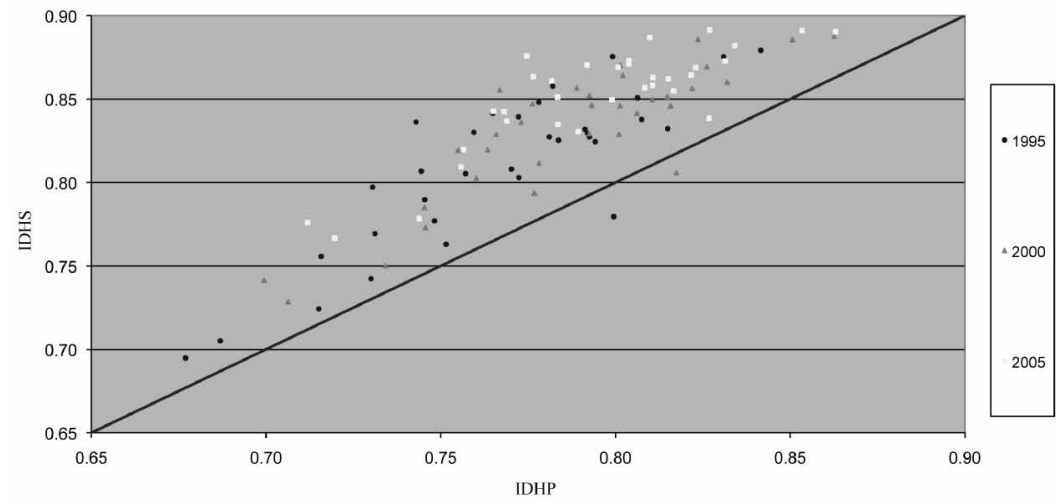

Fuente: Cálculos propios con base en INEGI (1990, 1995, 2000, 2005).

\section{Figura VIII}

\section{IDHS $1995 v s$. incremento porcentual IDHS 1995-2000}

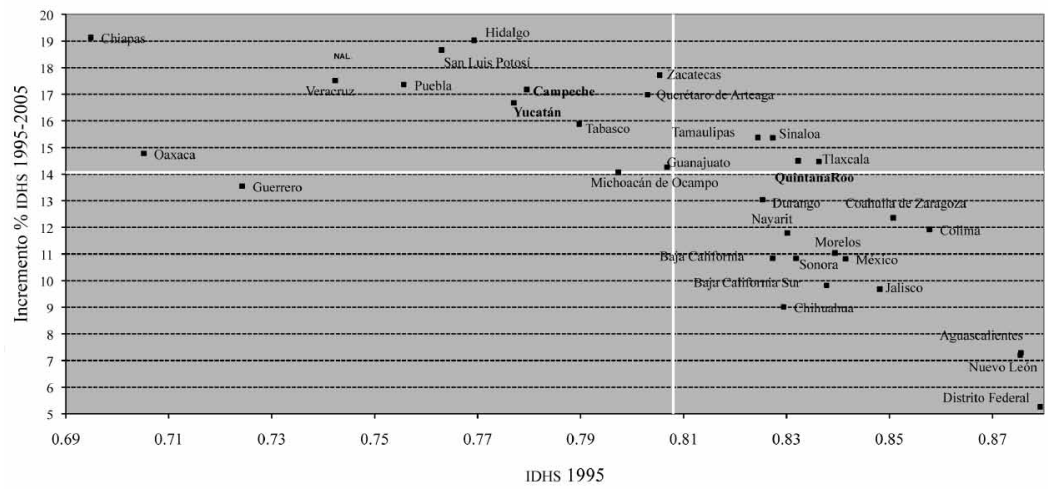

Fuente: Cálculos propios con base en INEGI (1990, 1995, 2000, 2005).

logrado los mayores índices; algo similar ocurre en Quintana Roo, donde Benito Juárez, que en 2005 albergaba a más de la mitad de la población estatal, es el principal centro turístico en México y durante el decenio tuvo el mayor IDHs. En Yucatán 82 de los 106 municipios tienen menos de 10,000 habitantes, donde Mérida es el municipio más poblado y uno de los de mayor DH en el estado.

\subsection{Equidad de género}

En el interior de cada población se originan diferencias en el desarrollo entre grupos, una de las más importantes y notorias es la que se da entre 


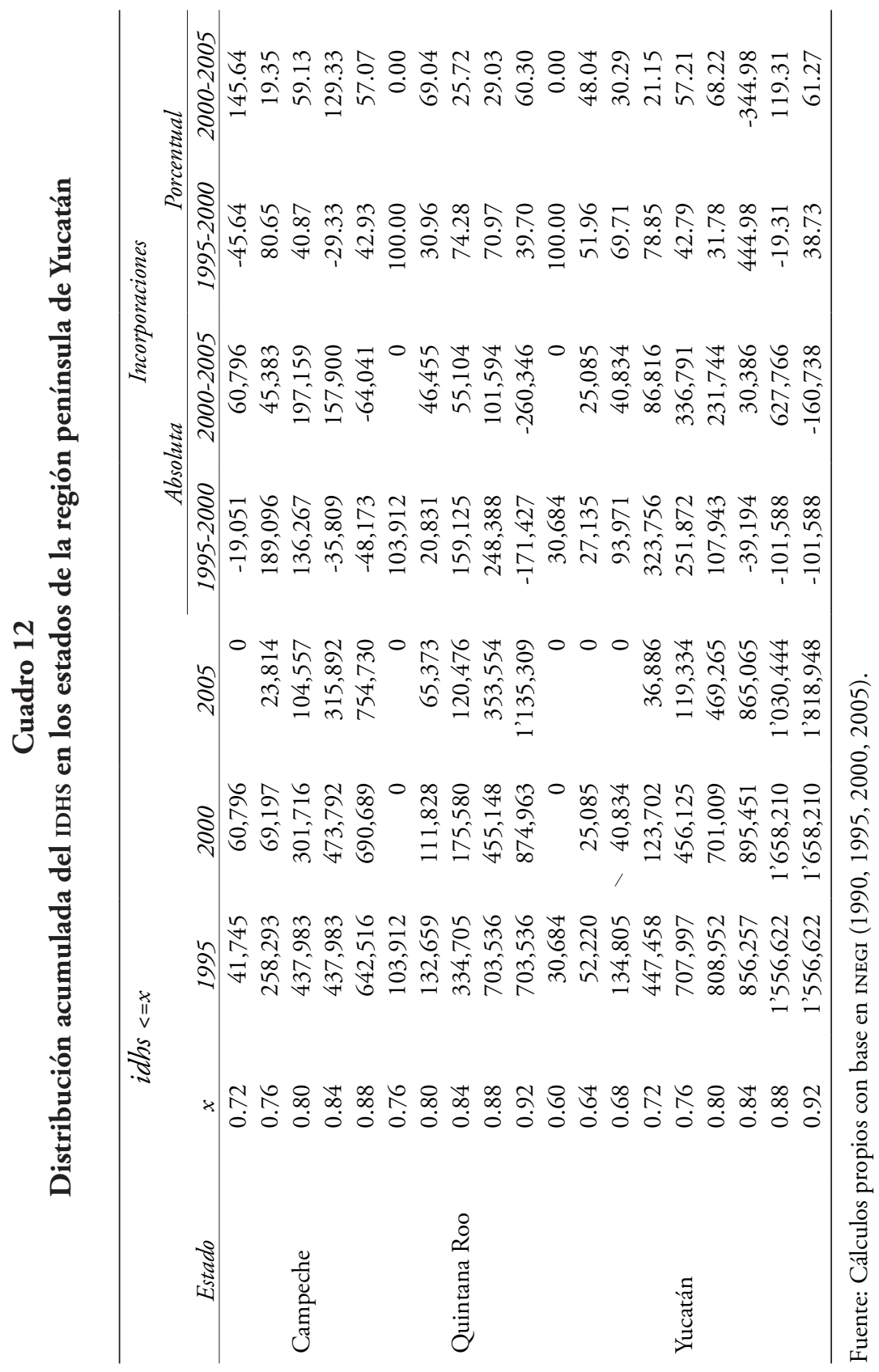


hombres y mujeres: el género femenino enfrenta situaciones de menor calidad de vida y menores oportunidades para poder competir en igualdad de condiciones en el sector laboral. En los últimos años se han dado avances hacia la equidad de género, pero la realidad es desalentadora, "La igualdad, en ocasiones, se ha conseguido en términos jurídicos, pero no reales. De modo que las mujeres ocupan menos cargos de responsabilidad, tanto en la política como en la economía, reciben salarios inferiores a los hombres en trabajos similares, les afecta en mayor medida el desempleo y cargan con la mayor parte del trabajo doméstico" (Berzosa, 2006: 1).

En México existen amplias discrepancias en las oportunidades que gozan hombres y mujeres, en la distribución del ingreso, en opciones productivas, en la generación de patrimonio y el acceso a protección social (Ibarrarán y Robles, 2003), las oportunidades para ambos géneros han aumentado con el paso del tiempo, pero estos crecimientos no han sido acordes con la situación de cada grupo, se ha reducido a menor ritmo la inequidad y como consecuencia no se ha logrado una plena ampliación de las oportunidades de la población en general.

Un método alternativo para determinar la magnitud de la inequidad de género en México y sus municipios consiste en realizar una prueba de diferencia de medias de dos poblaciones pareadas (ya que en el discurso, al estar hombres y mujeres en el mismo municipio, tienen las mismas condiciones), considerando el índice de desarrollo humano masculino $\left(\operatorname{IDHP}_{\mathrm{m}}\right)$ y el índice de desarrollo humano femenino $\left(\operatorname{IDHP}_{\mathrm{f}}\right)$ como dos variables aleatorias pareadas.

Es necesario aclarar que en este trabajo no se considera el DH como una variable aleatoria (va) sino como un derecho de la población, sin embargo el IDHP muestra propiedades similares a las de las va que permiten aplicar varios resultados de la teoría estadística.

$\mathrm{Si}\left(\mathrm{IDHP}_{\mathrm{m} 1,} \mathrm{IDHP}_{\mathrm{f1}}\right),\left(\mathrm{IDHP}_{\mathrm{m} 2}, \operatorname{IDHP}_{\mathrm{f} 2}\right), \ldots,\left(\mathrm{IDHP}_{\mathrm{m} 2454,} \mathrm{IDHP}_{\mathrm{f} 2454}\right)$, son las parejas de índices de DH masculino y femenino respectivamente, para cada uno de los 2,454 municipios que conformaban al país en 2005, y $\mathrm{d}_{\mathrm{i}}=\left(\right.$ IDHP $_{\text {mi }}-$ IDHP $\left._{\mathrm{f}}\right)$, bajo el supuesto de que las $\mathrm{d}_{\mathrm{i}}$ pueden ser explicadas por una distribución determinada con media $\mu_{D}$ y varianza $\sigma_{D}^{2}$, haciendo uso del teorema límite central, se puede probar la siguiente hipótesis estadística:

$$
H_{0}: \mu_{D}=0 \quad \text { versus } \quad H_{0}: \mu_{D} \neq 0
$$

La media y varianza muestrales son:

$$
\bar{d}=\frac{1}{2454} \sum_{\mathrm{i}=1}^{2454} d_{i}=0.3630 \text {, la cual estima a } \mu_{D} \mathrm{y}
$$


$s_{D}^{2}=\frac{1}{2453} \sum_{i=1}^{2454}\left(d_{i}-\bar{d}\right)^{2}=0.00098$, que estima a $\sigma_{D}^{2}$.

La estadística de prueba es:

$$
Z_{c}=\frac{\bar{d}-\mu_{D}}{\mathrm{~s}_{D} / \sqrt{2454}}=\frac{0.3630-\mu_{D}}{0.031305 / 49.5379}=\frac{0.3630}{0.00063194}=574.42
$$

El cual es muy superior a $Z_{\alpha / 2}=2.5758$, para $\alpha=0.01$, indicando que en México el promedio municipal de DH masculino es muy superior al femenino.

Particularizando el criterio anterior a las 32 entidades federativas de la República Mexicana, se observa una desigualdad de género muy significativa, desfavorable a las mujeres en todas ellas.

Cuadro 13

Determinación de la inequidad de género

\begin{tabular}{lcc}
\hline & $100^{*}(I D H P-I D G) / I D H P$ & $I D H P_{m}-I D H P_{f}$ \\
\hline Estados Unidos Mexicanos & 0.72802 & 0.02180 \\
Campeche & 0.66290 & 0.02702 \\
Quintana Roo & 0.61767 & 0.02455 \\
Yucatán & 0.47420 & 0.02194 \\
\hline
\end{tabular}

Fuente: Cálculos propios con base en INEGI (1990, 1995, 2000, 2005).

Por otro lado, calculando la diferencia porcentual entre el IDG y el IDHP respecto al IDHP para medir el grado de desigualdad de género en los municipios, si se considera una diferencia menor a $1 \%$ como no significativa, una diferencia mayor a $1 \%$ pero menor a $5 \%$ como significativa y una diferencia mayor a $5 \%$ como grave desigualdad de género, solamente Chiapas y Tabasco presentaron desigualdad de género significativa, las otras 30 entidades tienen diferencias no significativas.

Los dos criterios expuestos muestran conclusiones contradictorias, lo que indica que la métrica utilizada es determinante en los juicios que de ella se infieran. Por los resultados, es evidente que una diferencia de $1 \%$ entre el IDG y el IDHP significa, en términos de desarrollo, una enorme distancia que tal diferencia porcentual no exhibe. El problema radica en la construcción del IDG propuesto por el PNUD, puesto que al usar una aversión a la inequidad $\varepsilon=2$, prácticamente se requiere que las condiciones de hombres y mujeres sean muy diferentes para que el IDG lo capte. 
Para una explicación más detallada de los aspectos técnicos involucrados, consultar www.chapingo.mx/dicifo/demyc/idh/index.php.

\subsection{Variación e inequidad, 2005}

Para realizar este análisis se construyó un índice de inequidad (Inq) como uno menos el índice de Gini (InG) sobre el indicador de la variable de interés. El InG es uno de los índices más empleados en el estudio de la desigualdad, es una medida que resume la manera como se distribuye una variable entre los individuos de una población. A medida que el InG se acerca a uno, indica una concentración de la variable, y cuando se aproxima a cero revela una distribución dispersa, por lo que uno menos el InG actúa de manera contraria.

Para medir el grado de inequidad en la distribución territorial del desarrollo, se calcularon los índices de inequidad para cada uno de los factores del DH, InQInEsp, InqInedu, InqInpibp e Inqserv, así como para el IDHP (Inqidhp).

Si en una región no se concentran equitativamente las oportunidades para acceder a un buen nivel de salud, educación y cobertura de servicios básicos, se presentará una distribución territorial desigual del DH.

A escala nacional, la mayor inequidad se presentó en el PIBP, con un InqInpibp de 0.54471, ya que mientras el Distrito Federal tenía un Inpibp de 0.89917 con un PIBP de 21,861.61 dólares PPA; en el estado de Oaxaca el Inpibp fue de 0.60868 con un PIBP de 3,835.56 dólares PPA, la diferencia entre estas dos entidades fue abismal, 18,026.04 dólares PPA. El componente del desarrollo que reflejó la mayor equidad entre estados fue el Inele, con un InqInele de 0.14820, pues las 32 entidades de la República Mexicana presentaron valores de dicho indicador superiores a 0.90 , y la diferencia entre la cobertura de energía eléctrica que presentaron el Distrito Federal y Oaxaca, máximo y mínimo respectivamente, fue de sólo 6.64\% (98.53 y 91.89\%, respectivamente). En lo referente a la distribución de los servicios básicos, la cobertura de drenaje fue la que presentó la mayor desigualdad territorial en la República, esto debido a las diversas condiciones naturales y orográficas que se presentan a lo largo y ancho del país, desde el desierto y amplias planicies en el norte hasta la selva y topografías muy accidentadas en el sureste. En el cuadro 14 se presentan los estados en orden descendente, de acuerdo con el grado de inequidad medido a través del Inqidhp.

$\mathrm{Al}$ igual que en 2000, Baja California fue el estado más equitativo de la República Mexicana en 2005, lo cual significa que la variación del IDHP de un municipio a otro fue reducida, el mínimo IDHP en esta entidad fue de 0.803065 y el máximo de 0.815367 , la diferencia porcentual entre los 


\section{Cuadro 14 \\ Índice de inequidad del IDHP de los estados de la República Mexicana, 2005}

\begin{tabular}{lccrcc}
\hline \multicolumn{1}{c}{ Estado } & Inqidhp & Posición & \multicolumn{1}{c}{ Estado } & Inqidhp & Posición \\
\hline Baja California & 0.11311 & 1 & Colima & 0.16907 & 17 \\
Aguascalientes & 0.11976 & 2 & Zacatecas & 0.17389 & 18 \\
Sinaloa & 0.12998 & 3 & Sonora & 0.18673 & 19 \\
Durango & 0.13093 & 4 & Tlaxcala & 0.18759 & 20 \\
Chihuahua & 0.13763 & 5 & Yucatán & 0.19001 & 21 \\
Tamaulipas & 0.14156 & 6 & Querétaro & 0.19757 & 22 \\
Morelos & 0.1437 & 7 & Tabasco & 0.19988 & 23 \\
Baja California Sur & 0.14725 & 8 & San Luis Potosí & 0.20103 & 24 \\
Jalisco & 0.15218 & 9 & Michoacán & 0.20565 & 25 \\
Nayarit & 0.15625 & 10 & Puebla & 0.22629 & 26 \\
Guanajuato & 0.15827 & 11 & Campeche & 0.22847 & 27 \\
Quintana Roo & 0.16012 & 12 & Veracruz & 0.24832 & 28 \\
Nuevo León & 0.16143 & 13 & Hidalgo & 0.25914 & 29 \\
Distrito Federal & 0.16148 & 14 & Guerrero & 0.27712 & 30 \\
Coahuila & 0.16445 & 15 & Chiapas & 0.33869 & 31 \\
México & 0.16614 & 16 & Oaxaca & 0.37339 & 32 \\
\hline
\end{tabular}

Fuente: Cálculos propios con base en INEGI (1995, 2000, 2005) 1990.

valores extremos fue de $1.53 \%$. Es necesario mencionar que el estado está conformado por sólo cinco municipios, lo que coadyuva a una administración pública relativamente simple.

Nuevamente Oaxaca, el estado con el mayor número de municipios y la mayor diversidad cultural en el país, fue la entidad que presentó más inequidad. El estado albergaba a 3.40\% de la población nacional, estaba conformado por 570 municipios con grandes diferencias económicas, sociales y culturales, y sólo 14 tenían un DH clasificado como alto. Es el estado en el que se habla el mayor número de lenguas indígenas; todos los IDHP estuvieron entre los valores 0.50768 y 0.84281 , una diferencia de 66.01 por ciento.

En el estado de Campeche, la mayor inequidad entre sus municipios se presentó en la distribución del PIBP; en 2005 el municipio del Carmen aportó $53.67 \%$, Campeche $28.26 \%$, seguidos por Champotón con $4.66 \%$; Palizada, con sólo $0.42 \%$, fue el de menor aportación. Cabe mencionar que este último sólo contaba con 8,290 habitantes, mientras que los otros tres tenían poblaciones de 199,988, 238,850 y 76,116, respectivamente, es decir, la heterogénea distribución de la población estatal, característica en nuestro país, determina los enormes contrastes asociados a la actividad 
económica. La matrícula infantil presentó la menor inequidad entre los municipios campechanos, Candelaria tenía el menor porcentaje de habitantes entre seis y 14 años que asistían a la escuela con 79.91, mientras que el máximo se tuvo en la capital del estado, donde asistían a la escuela 88 de cada 100 personas en edad escolar, además dicha característica educativa mostró una mejor distribución territorial entre la población femenina.

Quintana Roo fue el estado de la península que presentó las mejores condiciones de DH. La menor equidad entre las localidades del estado se vio reflejada en el Indre (InqIndre de 0.28626), ya que mientras en Felipe Carrillo Puerto la cobertura de este servicio era solamente de $46.26 \%$, en Benito Juárez, municipio donde la cabecera municipal es Cancún, fue de $97.43 \%$. Las grandes diferencias se deben a la realidad económica de los distintos municipios, donde las mejores condiciones se tenían en localidades industrializadas o con gran actividad turística, a diferencia de los que basaban su economía en las actividades del sector primario. La mayor equidad se presentó en el Inele (InqInele de 0.13020), pues cada uno de los ocho municipios de la entidad tenía una cobertura del servicio de energía eléctrica superior a 90\%. En términos generales, en 2005 se presentaron para Quintana Roo condiciones de bienestar con una distribución bastante uniforme entre sus municipios.

En Yucatán, el estado con más municipios y población en la región, el InqIndre estatal fue de 0.62019; de los 106 municipios 57 tenían un Indre en la categoría baja, 42 en la categoría media y únicamente siete en el nivel alto. Además, el valor más bajo de este indicador fue de 0.08816 en la localidad de Mayapán que concentraba únicamente a 2,972 habitantes, es prácticamente rural, con agricultura como principal actividad económica, su orografía se clasifica como llanura de barrera con piso rocoso y cementado, alta y escarpada, lo cual dificulta la adecuación y construcción de la infraestructura requerida para expandir el servicio; mientras que en el municipio de Progreso, con más de 49,000 habitantes, se tenía una cobertura del servicio de drenaje de más de 95 por ciento.

\subsection{Curvas de calidad en la región península de Yucatán}

Las curvas de calidad municipal constituyen una técnica empleada en la clasificación de los municipios de un estado o región, con base en la eficiencia de éstos en la transformación del ingreso (PIBP en dólares PPA) en mejores condiciones de salud, educación y servicios públicos. Las curvas de calidad también permiten hacer comparaciones entre dos o más municipios con diferente PIBP trasladándolo al ingreso base del año de estudio para obtener un indicador cuantitativo de la efectividad y traducir el ingreso en desarrollo de un municipio con respecto a otro, a través del IDHS. 
En 2005 los estados de la península, Campeche, Quintana Roo y Yucatán, se ubicaron sobre las curvas de calidad 26, 20 y 19, respectivamente, mientras que el país se ubicó en la curva de calidad 22. Es decir, la calidad de estos tres estados, respecto a la del país, fue favorable para Yucatán y Quintana Roo, mientras que Campeche se muestra a la zaga, es decir, su manera de trasformar los ingresos en mejores condiciones de salud, educación y servicios no fue ni la promedio. Con respecto al Inpibp se localizaron en las posiciones tres, seis y 19, cada uno, esto debido en gran medida a la producción petrolera de Campeche y a las condiciones naturales que permiten que el turismo sea la principal actividad económica en Quintana Roo. Por otro lado, las condiciones de Yucatán eran muy diferentes ya que la mayoría de sus habitantes se encontraban en comunidades pequeñas dedicadas sobre todo a las actividades del sector primario y, en el caso de los municipios del sureste del estado, gran cantidad de sus habitantes se traslada a Quintana Roo a trabajar, además el tamaño de su población era mayor a la conjunta de los otros dos estados.

Los contrastes entre las parejas de valores PIBP e IDHS son ilustrativos del concepto de calidad definido en este trabajo. En 2005 el pIBp nacional fue de 8,998.34 dólares PPA, y el IDHs de 0.84968. En Quintana Roo el рIBP fue de 12,545.15 dólares PPA y el IDHS de 0.87274, mientras que en Yucatán se registró un PIBP de 7,167.17 dólares PPA, el más bajo de la región, a pesar de ello logró alcanzar un IDHs de 0.83483 . Nótese que el ingreso de Yucatán sólo representa 79.65\% del correspondiente al país, sin embargo, su IDHS representa $98.25 \%$. Es decir, los ingresos de la población de Yucatán fueron mejor empleados para mejorar las condiciones de vida de su población, que los del país en general. Una situación parecida ocurre con Quintana Roo, aunque en este caso el ingreso del estado y su condición de desarrollo es mayor que las del país. Campeche fue el estado que tradujo sus ingresos en bienestar de la manera menos adecuada. Tuvo un PIBP de 14,598.26 dólares PPA, el más alto de la región, sólo superado por el Distrito Federal y Nuevo León; aun así sólo logró alcanzar 0.83858 en su IDHS, el 24 en la escala nacional. La aparente situación contradictoria de Campeche, objetivada en un mayor ingreso pero un menor desarrollo, se ha puntualizado varias veces en el trabajo, pues contablemente tiene un ingreso desmesurado pero en la realidad, del mismo, es escaso lo que se incorpora a la entidad.

Presumiblemente debería existir una correspondencia más fuerte entre el ingreso y el DH de los habitantes como se presumía en el siglo pasado, cuando se introdujo el concepto de PIBP, sin embargo, la información con que se cuenta ahora exhibe la falacia de tal argumento, falacia potenciada por la economía de mercado, fincada en la ganancia y no en la racionalidad del consumo. 


\section{Figura IX}

\section{Calidad municipal en la región península de Yucatán}

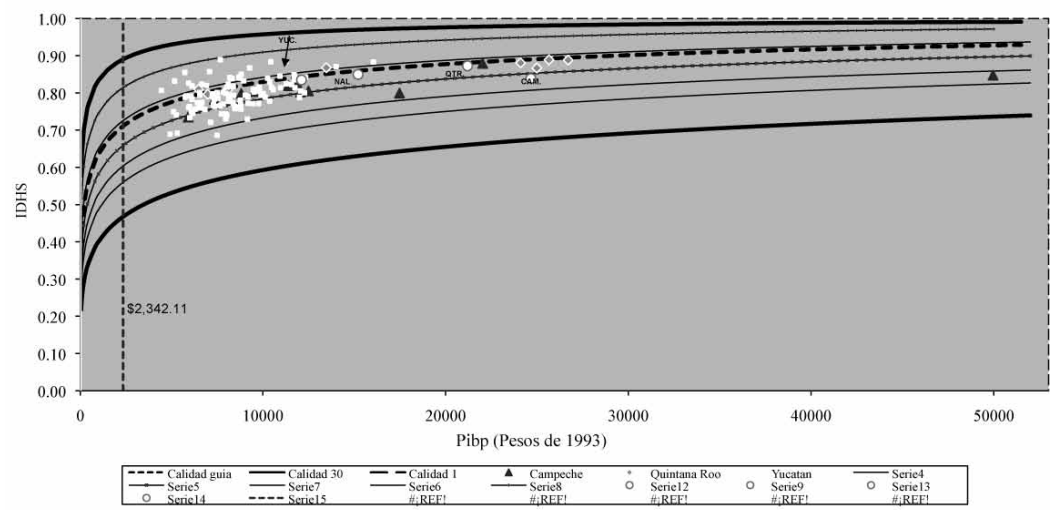

Fuente: Cálculos propios con base en INEGI (1990, 1995, 2000, 2005).

\section{Conclusiones}

Sin duda la precisión de los enunciados está en relación inversa con el tamaño del universo al que se refieren tales enunciados, entre mayor sea tal universo menor será la precisión que se alcance. Asimismo, si estos universos se refieren a conglomerados de seres vivos, no sólo se perderá precisión sino también los valores promedio serán medida unívoca de todos los miembros del universo que presentarán una dispersión respecto al mismo, la cual puede ser tan grande que la medida promedio resulte muy engańosa de las condiciones en las que están las partes del universo. Para esto último se introducen medidas de dispersión que ayudan a reflejar las condiciones de desigualdad en las que están los miembros de un conglomerado, del modo que se ha hecho en este trabajo. Para la imprecisión comentada al principio, lo conducente es el estudio de las partes que constituyen un todo, sin embargo el tratamiento de una región completa hace impráctica la posibilidad de analizar los detalles de las partes que constituyen a cada uno de los estados que la componen, por lo que las conclusiones que adelante se expresan deben verse en la gran escala que se manejan. Sin duda, los estudiosos de la región tendrán en este trabajo un referente que involucra la dinámica del DH en la península de Yucatán durante los últimos 15 años, y quedará por hacer todavía el análisis detallado de las situaciones particulares que muestren las abismales desigualdades existentes dentro de las no menos significativas aquí comentadas. 
El país vio incrementar su EV en 2.03 ańos entre 1995 y 2005, en gran medida por la disponibilidad y acceso a los servicios de salud pública, así como a las políticas educativas de prevención. El descenso en la mortalidad infantil, las variadas campañas de prevención de diabetes, obesidad, cáncer de mama y próstata, entre otras, el interés por la educación en la salud masculina, así como el mantenimiento a la derechohabiencia de los servicios de salud, son algunos de los factores que explican de tal incremento. ¿Cómo se puede calificar este avance? Una forma apropiada de hacerlo es compararlo con los avances que tuvieron otras naciones que en 1995 tenían una ev semejante a la de México. Sry Lanka (72.5), Seychelles (72), Venezuela (72.3), Barhéim (72.2), República Dominicana (73) y Argentina (72.60) tenían esperanzas de vida semejantes a las de México (72.58) en 1995 (PNUD, 1998); sus incrementos porcentuales durante el decenio fueron $0.03,-0.01,0.01,0.01,0.04,0.04$ y 0.03 , respectivamente, mientras que el incremento de México fue de 0.03 (PNUd, 2007-2008). Tales avances muestran que México lo hizo conforme a lo realizado en otros países de condiciones semejantes. No obstante, a esta tasa de avance los países mencionados tomarán muchas décadas para alcanzar la esperanza de vida de Japón en 2005, 81.25 años (PNUd, 2007-2008). Los estados de la región se han ajustado a la tendencia seguida por la mayoría de las otras entidades. Sin embargo se aprecia que en la península el estado de Yucatán es el que se muestra más rezagado, con las Ev más bajas, las mayores tasas de mortalidad y más deficiencias en infraestructura y personal médico, contrario a Quintana Roo.

En cuanto a educación, el país tuvo un incremento en su Inalf de más de $12 \%$ en el decenio, logrando superar el 0.90, es decir, en 2005 más de 90\% de los mexicanos mayores de 14 años fueron considerados como alfabetas. En la península, el estado de Quintana Roo mostró una mayor eficiencia en las medidas empleadas para cumplir el objetivo de reducir el analfabetismo, ya que a pesar de que al inicio del periodo tenía el mayor Inalf logró el mayor incremento porcentual del índice (18.97), además de alcanzar un indicador por arriba de 0.9. Dicho objetivo se vislumbra más complicado de cumplir en Yucatán por el tamaño de su población. En términos generales y de acuerdo con la unEsco, los estados de la región muestran una buena situación en cuanto a este indicador.

La matrícula infantil del país pasó de 86 a $88 \%$ durante el decenio. Los estados de la península se han mostrado lentos en este aspecto con respecto a las demás entidades, en 1995 sus Inmat eran inferiores al nacional, y para 2005 sólo Quintana Roo había superado dicha situación, sin embargo se ajustan claramente a la dinámica de la matrícula infantil del país. Aparentemente se contaba con los recursos necesarios para que la educación fuese de nivel razonable. De acuerdo con las pruebas Excale, 
realizadas por el Instituto Nacional para la Evaluación de la Educación (INEE), se observa en los resultados una correspondencia con los indicadores comentados. Para el sexto año de primaria en español sólo Quintana Roo (51.03\%) está por encima del promedio nacional (48.75\%) en porcentaje de aciertos, mientras que en matemáticas todos los estados de la península están por abajo del porcentaje de aciertos nacional (47.64). Algo semejante ocurre en el tercer año de secundaria, en español Quintana Roo $(51.58 \%)$ y Campeche $(51.88 \%)$ están por encima del promedio nacional (51.44\%), siendo Yucatán (50.16\%) el estado rezagado; en matemáticas todos están por abajo del promedio nacional (36.62\%), aunque ya muy cerca de éste (INEE, 2009a). Si a los datos anteriores se agrega la situación de México en la escala global, el atraso de los estados de la península es más notable. Baste mencionar el promedio nacional en lectura de las pruebas PISA (evaluación de competencias para la vida en jóvenes de 15 años en cualquier grado escolar desde primero de secundaria hasta el último del nivel medio superior), el cual es de 425 para el año 2009, por debajo del promedio de los países de la Organización para la Cooperación y el Desarrollo Económico (OCDE) que es de 493 (INEE, 2009b). Si en apariencia se tienen los recursos para la educación básica, en los resultados se nota un rezago considerable. El comparativo es importante porque está en el entorno del DH, donde la educación, como los otros factores, es vista en términos del desarrollo de capacidades, lo que se ve negado en las pruebas PISA.

En el aspecto económico se mantiene una polarización conocida y definida, donde los estados con las mejores condiciones se encuentran en el norte y los más desprotegidos están en el sur del país. En la República el incremento del pibp fue paupérrimo, consecuencia de un crecimiento importante en el primer quinquenio y un crecimiento nulo en el segundo. Yucatán mostró las mayores deficiencias en cuanto al Inpibp, posicionándose durante todo el periodo en el lugar 19 a escala nacional, mientras que Quintana Roo y Campeche, respectivamente, se ubicaron entre las primeras 10 posiciones. Por supuesto que este resultado es cuestionable en ambos estados y bajo la lente de las especificidades, por el lado de Quintana Roo el turismo genera los mayores ingresos, los cuales van a las corporaciones hoteleras y demás servicios, mientras que la extracción de petróleo en Campeche genera ingresos federales de los que pocos quedan en la entidad. El ingreso que finalmente llega a las personas es sin duda marginal, aun con ello, las diferencias que indican las mediciones quedan manifiestas en la información que los habitantes proporcionan en los censos y conteos; por ejemplo, las diferencias en ingresos estatales de los estados de la península, favorables a Quintana Roo y Campeche, se ven 
reflejadas en las diferencias que se observan en educación en estos estados, desfavorables a Yucatán.

El estado de Quintana Roo evidenció las mejores condiciones de DH en la región, y sobresalió del resto de los estados del país, teniendo uno de los cinco IDHP más altos durante el decenio, resultado del escenario existente en el estado en cuanto a cada uno de los componentes del desarrollo, a pesar de los desastres naturales sufridos. Por otro lado, Yucatán fue el único de los tres estados que para 2005 no se incorporaba a la categoría de DH alto.

En lo referente a los servicios, los estados de la región mostraron resultados mixtos. En la cobertura de agua Campeche va a la zaga y por debajo del promedio nacional; en energía eléctrica, por escasa diferencia, Yucatán tiene una mejor cobertura y todos ellos cerca del promedio nacional, y continúa siendo el drenaje el que muestra las peores condiciones en la región, en cierta medida por el tipo de suelo, sólo Quintana Roo ha logrado superar dicho problema, hasta cierto punto, dadas las necesidades del turismo. La expansión en la cobertura de cada uno de los servicios se ha dado a una mayor velocidad que el crecimiento de la población, reduciendo los rezagos existentes. Debe destacarse que la cobertura de servicios no muestra una correspondencia con el ingreso per cápita, por lo que Campeche y Quintana Roo tienen calidades más bajas que la de Yucatán, aunque Quintana Roo está muy cerca.

En los 32 estados de la República se cuenta con evidencias suficientes para concluir con un nivel de significancia de $1 \%$ que existe desigualdad de género significativa.

Si en una región no se distribuyen equitativamente las oportunidades de acceso a servicios de salud, educación, servicios básicos, etcétera, se presentará una distribución desigual del DH. En la región, para cada uno de los estados la mayor inequidad entre municipios se presentó en la distribución del ingreso, en gran medida por la heterogénea distribución territorial y por las diferentes actividades económicas prevalecientes en cada municipio, mientras que al igual que en todo el país, la mayor equidad la presentó el servicio de energía eléctrica.

Una conclusión integradora sobre la dinámica del DH en la península es que se observa un desarrollo favorable en Quintana Roo, mostrando poco a poco su inserción entre los estados con mejores opciones en el país. Los otros dos estados siguen la pauta ancestral seguida por el sur y no se ve que en un futuro cercano deje de repetirse la situación. En lo anterior se debe considerar que se habla de opciones minimas y que aun con las diferencias significativas que el trabajo muestra, las condiciones de los estados del país están lejos de las que se tienen en aquellos que muestran los desarrollos más avanzados. Por ello, los estados atrasados de la República requieren de políticas públicas importantes para su avance. 


\section{Bibliografía}

Anand, Sudhir y Amartya Sen (1995), "Gender Inequality in Human Development: Theories and Measurement, Human Development Report Office, undp, Occasional Paper 19, Nueva York, <http:// origin-hdr.undp.org/en/reports/global/hdr1995/papers/sudhir_ anand_amartya_sen.pdf>, 14 de noviembre de 2010.

Bassols-Batalla, Ángel (1994), "Regiones para el desarrollo de México", Problemas del Desarrollo, 96, Universidad Nacional Autónoma de México, México, pp. 51-63.

Berzosa, Carlos (2006), "Género y desigualdad", Semanario El Siglo de Europa, 687, Madrid, <http://www.elsiglodeuropa.es/siglo/ historico/2006/687/687econo.html>, 8 de julio de 2012.

Bracho, Teresa (1999), "Perfil educativo regional en México", Estudios Sociológicos, 53, El Colegio de México, México, pp. 703-742.

Castro-Soto, Gustavo (2007), "Radiografía de la electricidad en México", Edupaz/Mapder, Chiapas, http://www.omal.info/www/IMG/pdf/ RADIOGRAFIA_DE_LA_ELECTRICIDAD_EN_MEXICO. pdf, 25 de diciembre de 2010.

Conapo (Consejo Nacional de Población) (2000), "Vulnerabilidad sociodemográfica”, México, pp. 213-230, <http://www.conapo.gob. $\mathrm{mx} /$ publicaciones/sdm/Lapoblacion/13.pdf $>, 25$ de diciembre de 2010.

Conapo (Consejo Nacional de Población) (2001), Índices de desarrollo humano 2000, México, <http://www.conapo.gob.mx/publicaciones/indicesoc/IDH2000/desarrollo_humano.pdf>, 25 de diciembre de 2010.

Conapo (Consejo Nacional de Población) (2007), Principales causas de mortalidad en México 1980-2007, México, <http://www.conapo. gob.mx/publicaciones/mortalidad/Mortalidadxcausas_80_07. pdf>, 25 diciembre de 2010.

Demyc (Departamento de Estadística Matemática y Computo) (2003), Memoria técnica de los informes nacionales de desarrollo humano, Universidad Autónoma Chapingo, <www.chapingo.mx/dicifo/ demyc/idh/index.php>, 15 de mayo de 2008. 
De la Cruz, Carmen (2007), "Lo que no debemos olvidar del IDG y el IPG", documento de apoyo, curso Desarrollo Humano y Desigualdad de Género, escuela virtual, PNUD, Bogotá, pp. 1-3.

De la Torre, Rodolfo (1997), "Indicadores de desarrollo regional con información limitada”, en Gabriel Martínez, Pobreza y politica social en México, Fondo de Cultura Económica, México, pp. 273-295.

García-Verdú, Rodrigo (2002), "The Human Development Index and its Application to States in Mexico", Banco de México, México.

King, Gary (2006), "Evaluation of Seguro Popular: Baseline Analysis", Harvard University, Cambridge, <http://gking.harvard.edu/ gking/talks/spbaseP.pdf>, 13 de enero de 2011.

Ibarrarán, María Eugenia y Carlos Robles (2003), "Inequidad de género en desarrollo humano: el caso de México", pNud, México, <http://www.undp.org.mx/desarrollohumano/serie/images/ Cuadernos2003-07.pdf>, 10 de octubre de 2010.

INEGI (Instituto Nacional de Estadística, Geografía e Informática) (1990), XI Censo de Población y Vivienda, Banco de Información Económica (BIE) y Sistema Municipal de Bases de Datos (Simbad), INEgI, <http://www.inegi.gob.mx>, 10 de diciembre de 2010.

INEGI (Instituto Nacional de Estadística, Geografía e Informática) (1995), I Conteo de Población y Vivienda 1995, Banco de Información Económica (BIE) y Sistema Municipal de Bases de Datos (Simbad), INEGI, <http://www.inegi.gob.mx>, 11 de octubre de 2010.

INEGI (Instituto Nacional de Estadística, Geografía e Informática) (2000), XII Censo de Población y Vivienda, Banco de Información Económica (BIE) y Sistema Municipal de Bases de Datos (Simbad), INEGI, <http://www.inegi.gob.mx>, 11 de octubre de 2010.

INEGI (Instituto Nacional de Estadística, Geografía e Informática) (2001a), Anuario estadístico del estado de Campeche, INEGI, <http://www. inegi.org.mx/est/contenidos/espanol/sistemas/aee01/estatal/cam/ index.htm>, 12 de noviembre de 2010.

INEGI (Instituto Nacional de Estadística, Geografía e Informática) (2001b), Anuario estadístico del estado de Quintana Roo, INEGI, <http:// 
www.inegi.gob.mx/est/contenidos/espanol/sistemas/aee01/esta$\mathrm{tal} / \mathrm{qtr} /$ index.htm>, 12 de noviembre de 2010.

INEGI (Instituto Nacional de Estadística, Geografía e Informática) (2001c), Anuario estadístico del estado de Yucatán, INEGI, <http://www.inegi. gob.mx/est/contenidos/espanol/sistemas/aee01/estatal/yuc/index. htm>, 12 de noviembre de 2010.

INEGI (Instituto Nacional de Estadística, Geografía e Informática) (2002), Estadísticas a propósito del día internacional de la alfabetización. Datos nacionales, INEGI, <http://www.inegi.gob.mx/inegi/contenidos/espanol/prensa/contenidos/estadisticas/2002/alfabeti02. pdf>, 18 de mayo de 2008 .

INEGI (Instituto Nacional de Estadística, Geografía e Informática) (2005), II Conteo de Población y Vivienda 2005, Banco de Información Económica (BIE) y Sistema Municipal de Bases de Datos (Simbad), INEGI, <http://www.inegi.gob.mx>, 11 de octubre de 2010.

INEGI (Instituto Nacional de Estadística, Geografía e Informática) (2006a), Instituto Nacional de Estadística y Geografía, INEGI, <http:// www.inegi.org.mx/est/contenidos/espanol/sistemas/aee06/estatal/ cam/index.htm>, 12 de noviembre de 2010 .

INEGI (Instituto Nacional de Estadística, Geografía e Informática) (2006b), Anuario estadístico del estado de Quintana Roo, INEGI, <http:// www.inegi.gob.mx/est/contenidos/espanol/sistemas/aee06/esta$\mathrm{tal} / \mathrm{qtr} /$ index.htm>, 12 de noviembre de 2010.

INEGI (Instituto Nacional de Estadística, Geografía e Informática) (2006c), Anuario estadístico del estado de Yucatán, INEGI, <http://www.inegi. gob.mx/est/contenidos/espanol/sistemas/aee06/estatal/yuc/index. htm>, 12 de noviembre de 2010 .

INEGI (Instituto Nacional de Estadística, Geografía e Informática) (2010), Tasa der crecimiento media anual de la población por entidad federativa, 1990 a 2010, inegi, <http://www.inegi.org.mx/sistemas/sisept/Default.aspx?t=mdemo09\&s=est\&c=17511.

INEE (Instituto Nacional para la Evaluación de la Educación) (2009a), Presentación de resultados de la prueba pisa 2009, INEE, <http:// www.inee.edu.mx/>, 14 de noviembre de 2010. 
INEE (Instituto Nacional para la Evaluación de la Educación) (2009b), Explorador del Excale, INEE, <http://www.inee.edu.mx/explorador/>, 14 de noviembre de 2010.

Jarque, Carlos M. y Fernando Medina (1998), "Índices de desarrollo humano en México 1960-1990”, CEPAL, Santiago de Chile.

Kliksberg, Bernardo (2000), "América Latina: una región en riesgo. Pobreza, inequidad e institucionalidad social", Economía y Sociedad, 14, Universidad Michoacana de San Nicolás de Hidalgo, México, pp. 5-33.

PNUD (Programa de las Naciones Unidas para el Desarrollo) (1990), Informe sobre desarrollo humano 1990, Oxford University Press, Nueva York.

PNUD (Programa de las Naciones Unidas para el Desarrollo) (1998), Informe sobre desarrollo humano 1998. Cambiar las pautas actuales de consumo para el desarrollo humano del futuro, PNUD, Nueva York, <http://hdr.undp.org/en/media/hdr_1998_es_indicadores1.pdf $>, 27$ de diciembre de 2010.

PNUD (Programa de las Naciones Unidas para el Desarrollo) (2007-2008), Informe sobre desarrollo humano 2007-2008. La lucha contra el cambio climático: solidaridad frente a un mundo dividido, PNUD, Nueva York, <http://hdr.undp.org/en/media/HDR_20072008_ SP_Complete.pdf $>, 27$ de diciembre de 2010.

Ramírez-Magaña, Alejandro (1999), "Índice de desarrollo humano del estado de Guanajuato", Revista del Centro de Desarrollo Humano de Guanajuato, A.C., 3, Centro de Desarrollo Humano de Guanajuato, Guanajuato, pp. 9-28.

Scott, John (2006), "Seguro Popular Incidence Analysis", en Decentralize Service Delivery for the Poor, Banco Mundial, Washington, pp. 147-165.

Sen, Amartya (2000), Desarrollo y libertad, Planeta, Barcelona.

SEP (Secretaría de Educación Pública) (2006-2007), Principales cifras, sistema educativo de los Estados Unidos Mexicanos, SEP, México, <http://www.dgpp.sep.gob.mx/principalescifras/>, 25 de octubre de 2010. 
ss (Secretaría de Salud) (2005), Defunciones y mortalidad general, <http:// sinais.salud.gob.mx/descargas/xls/m_002.xls $>, 6$ de julio de 2012.

ss (Secretaría de Salud) (2006), Indicadores de recursos materiales y humanos, <http://sinais.salud.gob.mx/descargas/xls/rfmyh_ssa_i_001. xls>, 15 de enero de 2011.

Tun-Dzul Fernando y Heyden Manzanilla-Domínguez (2005), "Los huracanes: su impacto en la península de Yucatán en los últimos 100 años", El Colegio de la Frontera Sur, Chetumal, <http:// quintanaroo.webnode.es/news/impacto-de-huracanes-en-el-ultimo-siglo-/>, 6 de enero de 2011.

Zamudio-Sánchez, Francisco José, Mónica Pérez Miranda y Carlos Vargas Tames (2001), Primer informe sobre desarrollo humano en México 1995, Universidad Autónoma Chapingo-Centro de Desarrollo Humano de Guanajuato, A.C., Guanajuato.

Zamudio-Sánchez, Francisco José, Mónica Pérez Miranda y Carlos Vargas Tames (2002), Segundo informe sobre desarrollo humano de México: análisis comparativo del desarrollo humano en el lustro 19952000, Universidad Autónoma Chapingo-Centro de Desarrollo Humano de Guanajuato, A.C., Guanajuato.

Recibido: 6 de noviembre de 2008. Reenviado: 20 de enero de 2011. Aceptado: 22 de marzo de 2011.

Francisco José Zamudio-Sánchez. Es Ph. D. con mayor en estadística por la Universidad Estatal de Iowa. Actualmente labora en el Departamento de Estadística, Matemática y Cómputo, de la División de Ciencias Forestales. Es director del Programa Nacional sobre Desarrollo Humano en México de la Universidad Autónoma Chapingo. Línea de investigación actual: modelos lineales y estadística social. Entre sus publicaciones destacan: en coautoría, "Análisis comparativo del desarrollo humano en los estados de Chihuahua y Sinaloa, 1995-2000", Región y Sociedad, xv (35), El Colegio de Sonora, Hermosillo, pp. 43-73 (2006); "El desarrollo humano en el Estado de México y el Distrito Federal, 1995-2000: una lejana vecindad”, Economía, Sociedad y Territorio, viI (25), El Colegio Mexiquense, Zinacantepec, pp. 65-102 (2008); "Un índice de ruralidad para México", Espiral: Estudios sobre Estado y Sociedad, xIV (42), Univer- 
sidad de Guadalajara, Guadalajara, pp. 179-214 (2008); "Desarrollo humano en el estado de Oaxaca, 1995-2000", Secuencia, 72, Instituto Mora, México, pp. 111-136 (2008).

Margarito Soriano-Montero. Es maestro en ciencias por el Instituto de Matemáticas Aplicadas y Sistemas de la Universidad Nacional Autónoma de México (unam). Actualmente labora en el Departamento de Preparatoria Agrícola de la Universidad Autónoma Chapingo. Es responsable de las estadísticas del Programa Nacional sobre Desarrollo Humano en México de la Universidad Autónoma Chapingo 2002-2004. Su línea de investigación actual es: confiabilidad.

Pedro Ibarra-Contreras. Es licenciado en estadística por la Universidad Autónoma Chapingo. Actualmente labora en la Dirección General de Investigación Económica del Banco de México. Su línea de investigación actual: organización industrial y economía laboral. 